\title{
Variable branching characteristics of peripheral taste neurons indicates differential convergence
}

Abbreviated title: Variation in Taste Neuron Morphology

Tao Huang $^{1 *}$, Lisa C. Ohman ${ }^{1 *}$, Anna V. Clements ${ }^{1}$, Zachary D. Whiddon ${ }^{1}$, and Robin F. $\mathrm{Krimm}^{1 \#}$

${ }^{1}$ Department of Anatomical Sciences and Neurobiology, University of Louisville School of Medicine, Louisville, KY 40202

*These authors contributed equally to this work

\#Correspondence to: Dr. Robin Krimm, robin.krimm@louisville.edu, MDR building, room 405B, 511 S. Floyd Street, Louisville, KY 40202

Key Words: taste, geniculate neuron, morphology, afferent, tongue

Classification: Biological Sciences

Number of pages: 37

Number. of Figures: 9

Number of Tables: 0

Number of words in abstract: 235

Number of words in introduction: 634

Number of words in discussion: 1456

Acknowledgements: We would like to thank Chad Samuelsen for his help with cluster analysis and comments on the manuscript. We would like to thank Kaytee Horn for her help breeding and genotyping the mice and additional technical assistance. This project was supported by R21 DC014857 and R01 DC007176 to R.F.K and F31 DC017660 to L.O. 


\section{ABSTRACT}

Taste neurons are functionally and molecularly diverse, but their morphological diversity remains completely unexplored. Using sparse cell genetic labeling, we provide the first reconstructions of peripheral taste neurons. The branching characteristics across 96 taste neurons show surprising diversity in their complexities. Individual neurons had 1 to 17 separate terminal arbors entering between 1 to 7 taste buds, 18 of these neurons also innervated non-taste epithelia. Axon branching characteristics are similar in gustatory neurons from male and female mice. Cluster analysis separated the neurons into four groups according to branch complexity. The primary difference between clusters was the amount of the nerve fiber within the taste bud available to contact taste-transducing cells. Consistently, we found that the maximum number of tastetransducing cells capable of providing convergent input onto individual gustatory neurons varied with a range of 1-22 taste-transducing cells. Differences in branching characteristics across neurons indicate that some neurons likely receive input from a larger number of taste-transducing cells than other neurons (differential convergence). By dividing neurons into two groups based on the type of taste-transducing cell most contacted, we found that neurons contacting primarily sour transducing cells were more heavily branched than those contacting primarily sweet/bitter transducing taste cells. This suggests that neuron morphologies may differ across functional taste quality. However, the considerable remaining variability within each group also suggests differential convergence within each functional taste quality. Each possibility has functional implications for the system. 
Significance statement: Taste neurons are considered relay cells, communicating information from taste-transducing cells to the brain, without variation in morphology. By reconstructing peripheral taste neuron morphologies for the first time, we found that some peripheral gustatory neurons are simply branched, and can receive input from only a few taste-transducing cells. Other taste neurons are heavily branched, contacting many more taste-transducing cells than simply branched neurons. Based on the type of receptor cell contacted, branching characteristics are predicted to differ across (and within) quality types (sweet/bitter vs sour). Therefore, functional differences between neurons likely depends on the number of taste-transducing cells providing input and not just the type of cell providing input. 


\section{Introduction}

The chemical information from food is detected by cells in the taste bud (taste-transducing cells) and carried to the brain by sensory neurons. This chemical information includes taste qualities described as sweet, salty, sour, bitter, and umami (Contreras and Lundy 2000, Spector and Travers 2005, Yarmolinsky, Zuker et al. 2009, Carleton, Accolla et al. 2010, Ohla, Yoshida et al. 2019) as well as those that are not easily classified (Bachmanov, Tordoff et al. 1996, Tordoff 2001, Liu, Archer et al. 2016, Tordoff 2017, Lim and Pullicin 2019). Moreover, taste stimuli vary in intensity, and differences in stimulus intensity impacts quality coding (Ganchrow and Erickson 1970, Wu, Dvoryanchikov et al. 2015). Adding to their functional diversity, taste neurons respond to and are modulated by somatosensory stimuli (Lundy and Contreras 1997, Breza, Curtis et al. 2006, Yokota and Bradley 2016, Yokota and Bradley 2017). While numerous studies have focused on the functional diversity of this population of neurons, only recently has there been confirmation of their molecular diversity (Dvoryanchikov, Hernandez et al. 2017, Zhang, Jin et al. 2019). However, their morphological diversity remains completely unexplored.

Unlike the sensory neurons of skin (Maksimovic, Nakatani et al. 2014, Castillo, DiazFranulic et al. 2018), taste neurons do not participate in transduction (Chandrashekar, Hoon et al. 2006). Therefore, functional differences between gustatory neurons are thought to be determined entirely by the type of taste-transducing cell they innervate (Yarmolinsky, Zuker et al. 2009). Consistent with this hypothesis, retrograde tracing studies conclude that most taste neurons innervate only a single taste bud (Zaidi and Whitehead 2006). In addition, functional studies conclude that limited convergence is required for quality coding at mid-range stimulus concentrations (Yoshida, Yasumatsu et al. 2006). Despite the functional and molecular diversity of taste neurons, anatomical variation in these neurons was not expected, and was difficult to measure.

A definitive measure of morphological diversity necessitates full reconstruction of the peripheral axon of individual neurons. However, anatomical tracers do not travel the full length of 
peripheral taste axons. Genetic labeling provides a practical alternative (Wu, Williams et al. 2012, Bai, Lehnert et al. 2015, Kuehn, Meltzer et al. 2019); however, obstacles to this approach also exist. First, the number of taste neurons innervating the tongue is miniscule relative to the somatosensory innervation of lingual epithelium. Thus, if neurons from both populations are labeled, individual taste axons will be indistinguishable from somatosensory axons. As these two populations of neurons express many of the same factors, the majority of genetic labels used in other systems are impractical for reconstructing taste neurons (Wu, Williams et al. 2012). Lastly, the tongue is an unusually dense tissue requiring relatively thin sections for antibody labeling, which renders full neuron reconstruction unusually tedious.

It is important to overcome these practical limitations to fully reconstruct the axons of taste neurons for several reasons. Classification of morphological diversity can be combined with molecular expression data to correlate morphologies with distinct functions (Abraira and Ginty 2013). Knowing whether morphological differences are present dictates whether this direction of inquiry would be informative for the field of taste. Furthermore, the degree of branching likely reflects the degree of connectivity with taste-transducing cells (Chklovskii 2004), which would indicate that gustatory neuron responses are determined by both the type and number of transducing cells providing input.

In the present study, we used genetically directed sparse-cell labeling to fully reconstruct the complete peripheral morphologies of 96 neurons innervating taste buds in the tongue. Quantitative analyses revealed a surprising degree of diversity between taste neurons that, on average, innervate more taste buds than previously estimated. We demonstrate that neurons with more complex axonal arbors contact a large number of taste-transducing cells, whereas those with simple endings contact only a few taste-transducing cells. We conclude that individual neurons likely receive differing amounts of convergent information from taste-transducing cells. 


\section{MATERIALS AND METHODS}

\section{Animals}

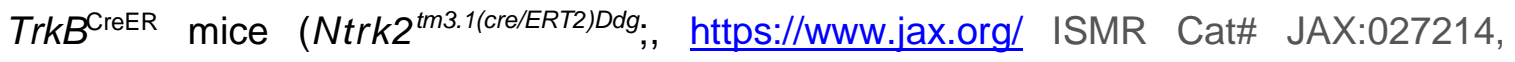
RRID:IMSR_JAX:027214) were crossed with Cre-dependent alkaline phosphatase (AP) mice (https://www.jax.org/; IMSR Cat\# JAX:009253, RRID:IMSR_JAX:009253) to obtain TrkB ${ }^{\text {CreER:AP }}$ mice, or with Cre-dependent tdTomato mice ((Rutlin, Ho et al. 2014) https://www.jax.org/ RRID:

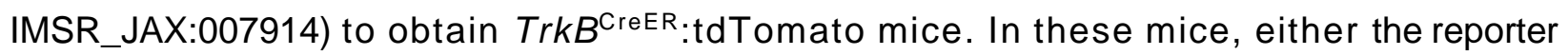
gene AP or tdTomato is expressed following TrkB-driven Cre-mediated gene recombination.

Fungiform taste buds decrease in number postnatally (Liebl, Mbiene et al. 1999, Patel and Krimm 2012), such that varying the experimental age of examination could introduce variation in fiber morphology. To avoid this potential confounding variable, all fibers were reconstructed in mice at postnatal day 60-62. Although all animals were examined at the same age, 27 TrkB ${ }^{\text {CreER }: A P ~ m i c e ~ w e r e ~ i n j e c t e d ~ w i t h ~ t a m o x i f e n ~}(0.5 \mathrm{mg}-1.0 \mathrm{mg})$ at $\mathrm{P} 40$, axons were reconstructed from 17 of these 27 mice ( 8 male and 9 female), while 10 either had no labeled axons or too many labeled axons to reconstruct (more than 4). To select from all neurons expressing TrkB during development, 48 pregnant dams were injected with 4 hydroxy tamoxifen at E15.5, 26 litters survived through birth, producing 21 mice ( 9 male an 12 female) that were of the correct genotype and had the correct number of axons to permit reconstruction (1-4), 115 mice either had 0 labeled axons or too many to accurately reconstruct (more than 4). To avoid selections bias, either none of the fibers were reconstructed or all of the fibers were reconstructed for each half tongue. In order to label one single tdTomato-positive axon in the tongue, 94 TrkB ${ }^{\mathrm{CreER}}$ :tdTomato mice were injected with tamoxifen $(0.3 \mathrm{mg}-1 \mathrm{mg})$, producing a total of 21 half tongues with one labeled axon. The remaining 167 tongue halves were determined to have either no labeled axons or too many (more than one) following serial sectioning of the tongue muscle. All animals were cared for in accordance with the guidelines set by the U.S. Public Health Service Policy on the Humane 
Care and Use of Laboratory Animals and the NIH Guide for the Care and Use of Laboratory Animals.

\section{Tamoxifen injections}

Tamoxifen (T-5648, Sigma, St. Louis, MO) was dissolved in corn oil (C-8267, Sigma, St. Louis, MO) at $20 \mathrm{mg} / \mathrm{ml}$ by shaking and heating at $42^{\circ} \mathrm{C}$ and injected at P40. For embryonic injections, 4-Hydroxytamoxifen (4-HOT) (H-7904, Sigma, St. Louis, MO), the active metabolite of tamoxifen, was injected intraperitoneally into pregnant dams at E15.5. 4-HOT was prepared and injected as previously reported (Wu, Williams et al. 2012). Briefly, 4-HOT was dissolved in ethanol at $20 \mathrm{mg} / \mathrm{ml}$ by shaking and incubating at $37^{\circ} \mathrm{C}$ for $15 \mathrm{~min}$ and then stored at $-20^{\circ} \mathrm{C}$. Before use, the stock solution was dissolved in sunflower seed oil to a final concentration of $10 \mathrm{mg} / \mathrm{ml} 4-\mathrm{HOT}$, and the ethanol was evaporated by centrifugation under vacuum.

\section{Alkaline phosphatase staining}

Mouse tongues were dissected following perfusion with PBS containing $2 \%$ paraformaldehyde (PFA), $0.5 \%$ glutaraldehyde, and $2 \mathrm{mM} \mathrm{MgCl}_{2}$. The tongues were cut in half and postfixed for $2 \mathrm{~h}$. After rinsing twice in PBS with $2 \mathrm{mM} \mathrm{MgCl}_{2}$, the tissues were transferred to PBS without $\mathrm{MgCl}_{2}$, and incubated at $65^{\circ} \mathrm{C}$ for $2 \mathrm{~h}$ to inactivate endogenous alkaline phosphatase (AP). Then, the tongues were frozen in O.C.T. (Sakura Finetek, USA) and stored at $-80^{\circ} \mathrm{C}$ before serial sectioning at $180 \mu \mathrm{m}$. The staining was performed according to a previous publication with slight modification (Badea, Wang et al. 2003). Briefly, the tongue sections were washed in $0.1 \mathrm{M}$ Tris- $\mathrm{HCl}(\mathrm{pH}$ 7.5) followed by $0.1 \mathrm{M}$ Tris- $\mathrm{HCl}(\mathrm{pH}$ 9.5) before incubating overnight at room temperature in 0.1 M Tris- $\mathrm{HCl}(\mathrm{pH} 9.5)$ containing nitroblue tetrazolium and 5-bromo-4-chloro-3indolyl-phosphate at the recommended concentrations (Vector Laboratories). After washing in PBS and postfixing in 4\% PFA, the stained tissues were dehydrated with an ethanol series and 
cleared and mounted with 2:1 benzyl benzoate/benzyl alcohol (Sigma); sections were sealed with DPX (VWR).

\section{Chorda tympani nerve transection}

Mice were sedated with a $0.32-\mathrm{mg} / \mathrm{kg}$ intramuscular injection of medetomidine hydrochloride (Domitor) and anesthetized with $40 \mathrm{mg} / \mathrm{kg}$ ketamine hydrochloride (Ketaset). Mice were placed in a nontraumatic head holder to provide access to the nerve in the neck via a ventral approach (Guagliardo and Hill 2007). The chorda tympani nerve was located as it bifurcates from the lingual branch of the trigeminal nerve and was transected (portion was removed) without damaging the trigeminal nerve. The wound was sutured, and mice recovered on a watercirculating heating pad before being returned to their home cages. Atipamezole hydrochloride ( 2 $\mathrm{mg} / \mathrm{kg}$ ) was injected intramuscularly immediately after surgery to promote the reversal of anesthesia and thus reduce recovery time. Meloxicam was also administered orally through food pellets for 2 days after surgery; to achieve a dose of $1-2 \mathrm{mg} / \mathrm{kg}, 0.5 \mathrm{cc}$ of a $0.5 \mathrm{mg} / \mathrm{ml}$ meloxicam solution was applied to each of two food pellets, which were then moistened with water and placed in the home cage. After 7 days, mice were euthanized and perfused with $4 \%$ PFA.

\section{Fluorescent anterograde nerve labeling}

The procedures used to label the chorda tympani with fluorescent tracers were previously

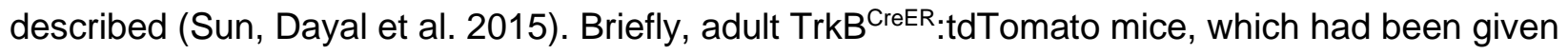
$4.0 \mathrm{mg}$ tamoxifen via gavage for 3 weeks, were anesthetized and placed in the head holder as described above. A water-circulating heating pad was used to maintain body temperature. The chorda tympani nerves within the right tympanic bulla were cut near and peripheral to the geniculate ganglion, and crystals of $3 \mathrm{kDa}$ fluorescein dextran (D3306; Invitrogen) were applied to the proximal cut end of the chorda tympani. A small amount of Kwik-Sil (World Precision Instruments, Inc., Sarasota, FL) was then placed over the cut ends of the nerves to prevent crystals from diffusing from the intended labeling site. Postsurgical treatment was the same as 
described above. After $24 \mathrm{~h}$, mice were euthanized and perfused with 4\% PFA. The geniculate ganglia were dissected and immediately mounted and imaged by confocal microscopy.

\section{Immunohistochemistry}

Mice were sacrificed by avertin overdose $(4 \mathrm{mg} / \mathrm{kg})$ and perfused transcardially with $4 \%$ PFA. Dissected tissues were postfixed in 4\% PFA for $2 \mathrm{~h}$ (for thin serial sections) or overnight (thick sections and whole mounts), rinsed with PBS, and transferred to $30 \%$ sucrose at $4^{\circ} \mathrm{C}$ overnight. A razor blade was used to remove the circumvallate papilla; the tongues were then carefully split down the midline with a razor blade under a dissection microscope. Tongues were frozen the next day in $\mathrm{OCT}$ and stored at $-80^{\circ} \mathrm{C}$ before sectioning on a cryostat or processing for whole-mount staining. To determine if tongue halves are innervated by a single fiber, the muscle at the base of the midline was isolated using a razor blade, frozen in OCT, and serially sectioned $(30 \mu \mathrm{m})$ on the cryostat. Serial sections were thaw mounted onto slides in order and then cover slipped with Fluoromount-G (SouthernBiotech, Birmingham, AL). Sections were examined under a fluorescence microscope to determine how many labeled fibers enter the tongue.

To visualize taste buds and their innervation in serial sagittal sections, each tongue half was sectioned sagittally from the midline to the lateral edge at $90 \mu \mathrm{m}$. Each section was processed in a separate well, so that the section order was maintained. Floating sections were blocked at $4^{\circ} \mathrm{C}$ in $0.1 \mathrm{M} \mathrm{PB}$ with $3 \%$ donkey serum and $0.5 \%$ Triton $\mathrm{X}-100$ overnight and then incubated for 5 days at $4^{\circ} \mathrm{C}$ with primary antibodies, rabbit anti-DsRed (1:500, RRID:AB_632496, Living Colors DsRed polyclonal; Takara Bio USA) and rat monoclonal anti-Troma1 (cytokeratin 8, 1:50, RRID:AB_531826; Developmental Studies Hybridoma Bank, lowa City, IA). Sections were then rinsed four times for 15 min each, incubated for 2 days in secondary antibodies, Alexa 488 antirat IgG (1:500; Jackson ImmunoResearch Labs Cat\# 712-545-153, RRID:AB_2340684) and Alexa 555 anti-rabbit IgG (1:500;Molecular Probes Cat\# A-31572, RRID:AB_162543), rinsed another four times for 15 min each, and then mounted and coverslipped. 
Whole-mount immunohistochemistry of the lingual epithelium was performed to visualize innervated taste buds. First, the underlying muscle and lamina propria were removed as described previously (Ohman-Gault, Huang et al. 2017). The isolated lingual epithelium was then washed for 15 min (3 times) in 0.1 M PB. Tissues were then incubated in blocking solution (3\% donkey serum, $0.5 \%$ Triton $\mathrm{X}-100$ in $0.1 \mathrm{MPB}$ ) at $4^{\circ} \mathrm{C}$ overnight and then incubated for 5 days at $4^{\circ} \mathrm{C}$ with primary antibodies (PLCß2, 1:500, RRID:AB_2630573; Santa Cruz Biotechnology) in antibody solution ( $0.5 \%$ Triton $\mathrm{X}-100$ in $0.1 \mathrm{M} \mathrm{PB})$. Tissues were rinsed four times for 15 min each with $0.1 \mathrm{M} \mathrm{PB}$, incubated with secondary antibodies (1:500, Alexa Fluor 488 AffiniPure, RRID:AB_2340619; Jackson ImmunoResearch), rinsed again (4 times for 15 min each with 0.1 $M P B)$, and then incubated with $5 \%$ normal rabbit serum in antibody solution. Tissues were then rinsed and incubated with AffiniPure Fab fragment donkey anti-rabbit $\lg \mathrm{G}(20 \mu \mathrm{g} / \mathrm{mL}$, RRID:AB_2340587; Jackson ImmunoResearch) in antibody solution, rinsed, and incubated with Zenon Alexa Fluor 555 rabbit IgG labeling kit (according to the instructions for Zenon complex formation [Z25305; Invitrogen]) using anti-DsRed (1:500; RRID:AB_10013483; Living Colors DsRed polyclonal; Takara Bio USA). Tissues were rinsed, incubated for 5 days at $4^{\circ} \mathrm{C}$ with Car4 primary antibody (1:500, RRID:AB_10013483; R\&D Systems), rinsed, and then incubated with secondary antibodies (1:500, Alexa Fluor 647 AffiniPure, RRID:AB_2340438). Tissues were then rinsed again, mounted with Fluoromount-G, and coverslipped (high precision, 0107242; Marienfeld).

\section{Confocal Imaging}

Taste bud images were obtained using an Olympus Fluoview FV1000 confocal laserscanning microscope with a $60 \times$ NA1.4 lens objective using a zoom of 3, Kalman 2. Image sizes were initially set at 1,024 × 1,024 pixels but were cropped to reduce scanning time and bleaching. Serial optical sections at intervals of $0.47 \mu \mathrm{m}$ in the $Z$ dimension were captured, which is the optimal size at $60 x$ magnification for 3D reconstruction. All colors were imaged sequentially in separate channels to avoid bleed through. Image stacks were then deconvolved using AutoQuant 
X3 software (Media Cybernetics, Maryland) to reduce out-of-focus florescence and improve image quality.

\section{Experimental Design and Statistical Analyses}

Image analysis

AP-stained taste axons were reconstructed under a microscope (using a 60x lens objective with a 2-mm working distance and numerical aperture of 1.0) using Neurolucida software (MBF Bioscience). The axons were reconstructed from where the axons enter the tongue to all terminal ends. Axons were only reconstructed from a tongue half when all the axons in the tongue half could be reconstructed. Typically, only half tongues with fewer than 4 labeled axons could be reconstructed. Thus, axons from half tongues with more than 4 labeled axons were not reconstructed. The tongue outline was reconstructed using Neurolucida and Photoshop software (Adobe). Individual arbors innervating whole immunostained taste buds were also reconstructed from confocal image stacks using Neurolucida software. Reconstructions were then analyzed using Neurolucida Explorer (MBF Bioscience).

Imaris software version 6.4.2 (Bitplane) was used to generate the 3D reconstructions and to measure proximity of nerve arbors to labeled taste cells. The colocalization function in Imaris was not used, because it contains many user-selected options that might contribute experimenter bias. More importantly, it was unclear how much colocalization there would be between labels in two separate cell types: the labeled taste bud cells and nerve arbors. Also, the physical relationship between any two florescent markers in a sample is influenced by tissue processing, intensity and wavelength of labels, the location of the labeled protein in the cells, deconvolution, orientation of the tissue, etc. Thus, the physical distance between nerve arbors and labeled taste bud cells (proximity) was measured with a distance transformation function in Imaris, which was previously described (Valm, Cohen et al. 2017). This algorithm permits identification of an object and then its distance from any/all other objects in a defined 3D space. This was accomplished 
using automated thresholding, which identified the surface of labeled objects (cells and nerve arbors) and then determined the distance between them in voxel increments. Thresholds were automatically generated with no input from the operator to limit bias. Because the sampling was at roughly twice the resolution of the microscope, distances of two voxels or less are equivalent to the colocalization artifacts that occur when two objects of different colors are sufficiently close (Corson and Erisir 2013, Stratford, Larson et al. 2017). However, the proximity analysis enables the distance between any two cells to be measured. To display the relationship between a single arbor and labeled taste bud cell, individual taste-transducing cells and terminal arbors were segmented in Imaris, and the fluorescent channel was only duplicated within the selected region. Segmentation was completed after analysis for illustrative purposes only.

\section{Data analysis.}

Sixteen different anatomical measures were used in the k-means cluster analysis to group neurons on the basis of similarity: age of the animal at the time of tamoxifen injection, number of taste buds innervated, total length of the peripheral axon, location in the tongue of innervated taste buds (back, middle, tip), number of branch points (nodes) below the epithelium, number of branch points (nodes) in the taste bud, total number of branch ends, number of branch ends per taste bud, mean terminal branch length, combined terminal branch length, combined terminal arbor length, number of terminal arbors, number of widened endings, number of non-taste endings, distance from the base of the tongue to the first branch point, and highest branch order (Fig. 1). Time of tamoxifen injection and sex of the animals were compared for each measure using either a Mann-Whitney $U$ test or t-test depending on whether the measure was normally distributed (tested with Shapiro-Wilk test). A Bonferroni correction factor for the number of tests (16) required a $p=0.003$ to statistical significance. Cluster analysis was performed using MATLAB (https://www.mathworks.com/help/stats/k-means-clustering.html?s tid=srchtitle). We tested 2 to 6 clusters and selected the 4-cluster model because it was the highest silhouette value with no 
negative values (second highest overall). Characteristics were compared across clusters by first testing whether the measure was normally distributed across all 4 clusters using the Shapiro-Wilk test. If the measure was normally distributed, then differences were determined using a one-way analysis of variance with a Bonferroni's post hoc analysis. If they were not normally distributed differences across multiple groups were compared using a Kruskal-Wallis test, while two groups were compared using a Mann-Whitney test. Multiple comparisons were avoided by comparing a limited number of factors relevant to the study. Because most of our measures were not normally distributed, Spearman correlations were used to analyze the relationship between variables. The alpha level was set at $p=0.05$, and actual $p$ values are reported. However, when more than one comparison was made for a measure, a Bonferroni's correction factor was used to adjust the alpha level.

\section{Results}

\section{Genetically directed sparse labeling of taste neurons.}

Taste neurons are functionally and molecularly diverse, but it is unclear whether there is any morphological diversity within this population of neurons. To evaluate morphological diversity, we used an inducible genetic technique that primarily labels taste neurons, and not the more abundant somatosensory neurons that innervate the tongue epithelium. The neurotrophin receptor TrkB is expressed in most neurons within the geniculate (taste) ganglion (Huang and Krimm 2010, Tang, Rios-Pilier et al. 2017, Rios-Pilier and Krimm 2019), but very few TrkB-positive trigeminal neurons innervate the tongue (Wu, Arris et al. 2018). Therefore, we used TrkB ${ }^{\text {CreER:alkaline phosphatase }(A P)}$ mice to reconstruct single taste nerve axons innervating the tongue (Rutlin, Ho et al. 2014). AP has been used to reconstruct single axonal arbors of somatosensory neurons (Badea, Williams et al. 2012, Wu, Williams et al. 2012), and it has the advantage of being stained with adequate intensity to be visualized in thick tissues, allowing the stained axons to be more readily reconstructed than with fluorescent labeling. 


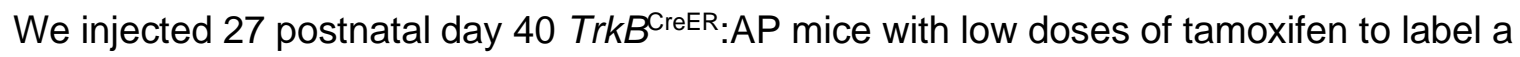
small number of individual neurons by adulthood (postnatal day 60) and reconstructed the peripheral axons from where the chorda-lingual nerve entered the tongue to where they terminated at the epithelial surface (Fig. 1A-E). The number of neurons labeled was variable and not strictly dose dependent. When more than four axons were labeled, they crossed paths, making it impossible to follow individual axons. To avoid the inherent bias of choosing the easiest to reconstruct axons when multiple axons were labeled, no reconstructions were completed from half tongues with more than four labeled axons. However, at the same doses of tamoxifen some tongues did not contain even a single labeled axon, while others had more than 4 labeled neurons. We reconstructed a total of 41 quantifiable neurons from 17 animals of the 27 injected with tamoxifen.

Since TrkB expression decreases in the geniculate ganglion after embryonic day 15.5 (E15.5) (Rios-Pilier and Krimm 2019), we were concerned that injections at postnatal day 40 did not include the full population of taste neurons. Therefore, we injected TrkB ${ }^{\text {CreER }}$ :tdTomato mice with a high dose of tamoxifen (4mg/day for two weeks) to capture as many TrkB-expressing neurons as possible and then labeled the taste neurons projecting to the tongue with a fluorescent tracer (Sun, Dayal et al. 2015). We found that $73 \%(n=2,71 \%, 75 \%)$ of tongue-innervating geniculate ganglion neurons were tdTomato-positive. Thus, the majority of TrkB-expressing taste neurons are labeled when adult TrkB ${ }^{\text {CreER }}$ :tdTomato mice are injected with tamoxifen (Rios-Pilier and Krimm 2019). Nevertheless, to circumvent the reduction of TrkB during development, we injected 58 pregnant dams (at E15.5) with $0.5,0.75$, or $1.0 \mathrm{mg} \mathrm{4-Hydroxytamoxifen.} \mathrm{A} \mathrm{total} \mathrm{of} 55$ axons were reconstructed from 21 of the 115 mice injected at E15.5. A total of 94 mice had either no labeled axons or more than 4 labeled axons, and so no axons were reconstructed from these mice. 
All reconstructed axons were from tongues of adult mice (postnatal day 60-62) regardless of the age at injection. The age at time of tamoxifen injection P40 $(n=41)$ and E15.5 $(n=55)$ had no significant effect $(\mathrm{p}<0.003$ with Bonferroni correction) on 16 measured morphological characteristics (listed in methods, Fig. $1 \mathrm{~F}-\mathrm{H}$, number of taste buds, $\mathrm{U}=1011, p=0.36$; total axon length, $\mathrm{t}_{(94)}=-0.69, p=0.49$; location in the tongue $\mathrm{U}=1097, \mathrm{p}=0.78$; number of branch points in the taste bud, $U=962, p=0.2$; number of branch ends, $U=798, p=0.01$; total number of branch ends, $U=1006, p=0.35$; branch ends per taste bud, $U=750, p=0.005$; combined terminal branch length, $\mathrm{U}=987, \mathrm{p}=0.28$; mean terminal branch length, $\mathrm{t}_{(94)}=-0 .-28, p=0.78$; number of terminal arbors, $U=968, p=0.22$; combined terminal arbor length, $U=960, p=0.2$; number of widened endings, $U=899, p=0.82$; length of axon from entry to first branch point, $U=812, p=0.02$; highest branch order, $U=1089, p=0.738$; number of non-taste endings, $U=1127, p=0.944$ ). There were no statistical differences ( $p<0.003$ with Bonferroni correction) in most of the morphological characteristics of axons reconstructed from male $(n=45)$ and female $(n=51)$ mice (number of taste buds, $U=885, p=0.05$; total axon length, $t_{(94)}=-0.42, p=0.69$; location in the tongue $U=1025$, $p=0.35$; the number of branch points below the epithelium (males $=2$ and females $4(U=792$, $p=0.007$ ) number of branch points in the taste bud, $U=1044, p=0.45$; number of branch ends, $U$ $=883, p=0.052$, number of branch ends per taste bud, $U=1111, p=0.74$; combined terminal branch length, $U=980, p=0.22$; mean terminal branch length, $t_{(94)}=-0.88, p=0.38$; number of terminal arbors, $U=812, p=0.013$; combined terminal arbor length, $U=953, p=0.15$; number of widened endings, $U=1131, p=0.91$; highest branch order, $U=820, p=0.015$; number of non-taste endings, $U=1073, p=0.425)$. The length of the axon from where it enters the tongue to the first branch point was slightly longer in males $(9.32 \mathrm{~mm})$ than females $(7.7 \mathrm{~mm} \cup=721, p=0.002)$, perhaps because the tongues were longer in males. Since differences based on age of tamoxifen injection and sex of the animal were minimal, we combined these data to yield a total of 96 fully reconstructed peripheral taste nerve axons (available to the research community at neuromorpho.org/dableFiles/tao krimm/Supplementary/Tao Krimm.zip). Across these neurons, 
we observed that individual axons had branch points in the tongue muscle, lamina propria, and epithelium (Fig. 1E). Branch points below the epithelium dictated the number of separate terminal arbors (Fig.1G), the portion of the axon innervating taste buds (range, 1-17 per neuron), whereas branch points inside the taste bud dictated the variation in complexity of these different terminal arbors.

The large number of branch points in the musculature and lamina propria was surprising, because in mice most taste neurons are thought to innervate only a single taste bud. Because these earlier studies assumed that gustatory neurons innervate adjacent taste buds, we examined heavily branched neurons to determine if they always innervated adjacent papillae. Many neurons innervate immediately adjacent papillae. However, we also found some taste neurons innervating multiple taste buds that were not located in the adjacent fungiform papillae in the tongue (Fig. 2A). Approximately $10 \%$ of the neurons had these wide anatomic receptive fields. Unfortunately, because sections were thick and tissue other than nerve fibers were unstained, it was not possible to determine if all of them had uninnervated papillae between innervated papillae. In addition, consistent with the finding that individual neurons tend to have anatomic receptive fields that spread out across the surface of the tongue, taste buds most often are innervated by only a single arbor from any individual neuron (Fig. 2B).

In the 38 tongues from which the 96 TrkB-positive axons innervating taste buds were reconstructed, we also observed 4 labeled axons that did not innervate taste buds. To determine if all labeled axons innervating the front of the tongue were from the primary taste nerve (the chorda tympani nerve), we injected $T r k B^{\text {CreER}}$ :tdTomato mice $(n=2)$ with a high dose of tamoxifen to label many TrkB-positive neurons and then transected the chorda tympani nerve. The axons remaining in the tongue after degeneration of the chorda tympani nerve are from another nongeniculate (taste) ganglion source (Guagliardo and Hill 2007). Although the number of TrkBpositive axons entering the tongue on the transected side was reduced compared to the 
contralateral side, some TrkB-positive axons remained in the tongue (Fig. 3A,B). These TrkBpositive axons innervating the tongue were likely from the trigeminal ganglion $(\mathrm{Wu}$, Arris et al. 2018). To determine if these non-chorda tympani TrkB-positive axons innervate taste buds, we quantified TrkB-positive innervation in the taste buds following unilateral chorda tympani nerve transaction on the intact side (Fig.3C,E) compared to the transected side (Fig.3D,F). We found that $100 \%$ of taste buds were innervated by tdTomato-labeled nerve axons on the intact side of the tongue, whereas only $5 \%$ of the taste buds were innervated by tdTomato-labeled nerve axons on the transected side. These innervated taste buds (four in two mice) were located at the midline ventral tongue tip where the midline is less clear, such that the innervation was likely from the contralateral chorda tympani nerve. We conclude that most TrkB-positive axons innervating the tongue are from the chorda tympani and that axons from other sources do not typically innervate taste buds. Therefore, axons that did not innervate taste buds were not reconstructed or included in the data set, as their nerve of origin was uncertain. So unfortunately, non-taste neurons within the chorda tympani (Dvoryanchikov, Hernandez et al. 2017, Yokota and Bradley 2017) nerve were not reconstructed.

\section{Individual axons of taste neurons vary in branching characteristics.}

Somatosensory neurons of the dorsal root ganglion can be classified as types by their distinct morphological endings in skin (Wu, Williams et al. 2012), this was not the case with taste neurons. However, we did observe that some taste axons branched very little, while others displayed extensive branching. To organize these neurons into descriptive groups (clusters), we measured 16 separate morphological characteristics (see Methods, Fig. 1F-H) and performed a k-means clustering analysis (Fig. 4). Silhouette values were used to estimate the optimal number of clusters. A four-cluster model provided the second highest mean silhouette value (Fig. 4A, 0.6944 ) while having no negative values, an indication of miss-assignment. We plotted histograms of all measured characteristics to determine which characteristics had the least amount of overlap 
between the clusters. The three characteristics allowing greatest separation between clusters are shown (Fig. 4B-D). These four clusters represented a gradual increase in branch complexity (measured morphological characteristics related to branching), from the simplest in cluster 1 to the most complex in cluster 4 (Fig. 4E). Just under half of the axons constituted cluster 1 . The remaining half were of increasing complexity, with the fewest in the most complex cluster (cluster 4). Combined terminal arbor length in the taste bud was the only measured trait with no overlap between clusters and provides the clearest separation of the clusters $\left(F_{(3,92)}=368, p=0.001\right.$; Fig. 4F). This measure corresponds to the amount of the nerve fiber (axon) available to contact tastetransducing cells. This finding implies that number and not just type of taste-transducing cells providing input to taste neurons varies across the taste neuron population.

\section{Nearly half $(44 \%)$ of taste axons have simple peripheral morphologies.}

Representative examples of the simple morphologies of two cluster 1 neurons are shown in Figure $5 \mathrm{~A}$ and $\mathrm{B}$, which innervated one and two taste buds, respectively. Terminal arbors (the portion of the axon inside the taste bud) of cluster 1 neurons typically had two (Fig. 5A) to three (Fig. 5B, second taste bud) branch points within the taste bud. Many terminal arbors branched within the taste bud, with only a single branch point and two branch ends. Most of the axons in cluster 1 neurons innervated one or two taste buds (81\%; Fig. $5 \mathrm{C}$ ), with only one to two terminal arbors (Fig. 5D). The more taste buds that are innervated the longer the axon $(\rho=0.68$, $p=0.0000002$, Fig. 5C). A smaller proportion (19\%) of the cluster 1 axons innervated three or more taste buds (Fig. 5C) with four or more separate terminal arbors (Fig. 5D). However, these terminal arbors were unusually short (Fig. 5E). Although cluster 1 neurons are morphologically simple, all but one branched at least once (Fig. 5F). All regions of the lingual epithelium (Fig. 1F) were innervated by cluster 1 neurons. Specifically, eight neurons innervated taste buds located at the tongue tip, twelve neurons spanned between the tip and the mid-region, eleven neurons 
innervated the tongue mid-region only, two neurons innervated the back of the tongue, and three neurons spanned between the mid-region and the back of the tongue.

\section{Complex taste axons innervate additional taste buds.}

Reconstructions of cluster 2 neurons illustrate an axon innervating three taste buds (Fig. 6A) and a second axon innervating seven taste buds (Fig. 6B). The neuron illustrated in Fig. 6A has widened endings (blue arrow), which were present in most taste nerve terminal arbors; a feature that did not differ across clusters. Although the function of these endings is not known, flattened contacts with taste-transducing cells or retraction bulbs are possibilities (Bernstein and Lichtman 1999, Zaidi, Cicchini et al. 2016). Compared to those in cluster 1, axons of cluster 2 neurons contained more branch points in the tongue muscle and lamina propria (median=1 $(\mathrm{N}=42)$ vs $4(\mathrm{~N}=36), \mathrm{U}=254, p=0.001)$ and innervated more taste buds (Fig. $6 \mathrm{C}$, median=2 $(\mathrm{N}=42)$ vs $3(\mathrm{~N}=36), \mathrm{U}=296, p=0.001)$; Thus, the entire axon innervating the tongue was longer on average for cluster 2 neurons than for cluster 1 neurons $\left(t_{(75)}=4.72, p=0.001\right.$, Fig. 6D). Similar to cluster 1 , most of the axons of cluster 2 neurons had a single terminal arbor innervating each taste bud (Fig. 6A and B). Cluster 1 and cluster 2 neurons did not differ in the number of branch ends per arbor (median=2 $(\mathrm{N}=42)$ vs $2(\mathrm{~N}=36)$, Mann-Whitney $\mathrm{U}=742, p=0.89)$ or terminal arbor length (median=20.8 $(\mathrm{N}=42)$ vs $24.6(\mathrm{~N}=36) \mu \mathrm{m}$, Mann-Whitney $\mathrm{U}=575, p=0.07)$. Therefore, the longer combined terminal arbor length of cluster 2 axons is primarily due to an increase in the number of taste buds innervated (Fig. 6C,E). There were cluster 2 neurons that innervated only 1 or 2 taste buds; however, these neurons had longer terminal arbors $(\rho=-0 .-94, p=0.0000002$, Fig. 6F). Like cluster 1, the axons of cluster 2 neurons innervated all regions of the lingual epithelium, but they were more likely to innervate multiple tongue regions. Specifically, axons branching to innervate both the tongue tip and the mid-region (16 axons) were the most common, with fewer axons exclusively innervating the tongue tip (9) or mid-region (4). In addition, five cluster 2 neurons innervated the back of the tongue, and one innervated both the back and the mid-region. 


\section{The most complex peripheral taste axons innervate taste buds with multiple terminal} arbors.

Representative examples of axons from cluster 3 (Fig. 7A) and cluster 4 (Fig. 7B) neurons illustrate their greater complexity. Cluster $3 / 4$ neurons innervate a similar number of taste buds as those in cluster 2 (Fig. $6 \mathrm{C}$, mean=3.53 $(\mathrm{N}=36)$ vs $4.22(\mathrm{~N}=18), \mathrm{t}=-1.445, p=0.154)$, but with significantly more terminal arbors (Fig. $7 \mathrm{C}$, median $=5(\mathrm{~N}=36)$ vs $8(\mathrm{~N}=18), \mathrm{U}=108, p=0.001)$. For example, seven taste buds were innervated by either 9 (Fig. 7A) or 10 (Fig. 7B) terminal arbors. Similar to these examples, clusters 3 and 4 combined had more arbors per taste bud than cluster 2 neurons (Fig. 7D, median=1.33 ( $N=36)$ vs $1.83(\mathrm{~N}=18), \mathrm{U}=173, p=0.006)$. Since the mean length of each arbor did not differ among the clusters $(H(2,93)=7.18, p=0.066)$, the combined length of the terminal arbors inside the taste bud for an axon is primarily determined by number of arbors $(\rho=0.75, p=0.0000002$, Fig. 7E). The only difference between neurons in clusters 3 and 4 is the combined lengths of the terminal arbors (Fig. 4F). Also, like axons of cluster 2 neurons, axons of cluster 3 and 4 neurons tended to innervate multiple tongue regions (9 out of 15).

All clusters had a few axons with branches ending outside of taste buds (Fig. 7A and F). Neurons with these "non-taste" endings typically have one or more branches that penetrate the epithelium, with a free nerve ending in a region of the tongue not occupied by a taste bud. In some cases, these are entirely different arbors than those innervating a taste bud, but they could also branch from a terminal arbor that innervates the taste bud. For example, the arbor of the first taste bud shown in Fig. 7A (dark blue) has a single branch arising from a branch point at the taste bud base (blue arrow) and extending into the lingual epithelium. A total of 18 neurons had these nontaste endings, which were most often in fungiform papillae (13 neurons) but also in filiform papillae (3 neurons) or both (2 neurons).

In summary, $44 \%$ of the peripheral axons of taste neurons had fairly simple branching characteristics (cluster 1), while the remaining 56\% showed more complex branching. There were no differences in the average lengths or number of branch ends of individual terminal arbors 
across the 4 clusters, indicating that combined arbor length is determined primarily by the number of arbors and not their size. Axons of cluster 2 neurons increased arbor number by innervating more taste buds than cluster 1 neurons. Whereas axons of cluster 3 and 4 neurons increased terminal arbor number by having more terminal arbors per taste bud.

\section{Each terminal arbor contacts a limited number of taste-transducing cells.}

Clusters were best separated by the combined terminal arbor length in taste buds, which is the amount of axon available to contact taste-transducing cells. Next, we sought to examine how many taste-transducing cells are contacted by a single neuron. Communication from tastetransducing cells to nerve arbors occur through both conventional synapses (Murray 1986, Kinnamon, Sherman et al. 1988, Yee, Yang et al. 2001) and release of ATP through CALHM1/3 channels (Taruno, Vingtdeux et al. 2013, Ma, Taruno et al. 2018, Romanov, Lasher et al. 2018). Unfortunately, it is not possible to observe these connections with light microscopy. However, an axonal arbor and taste-transducing cell must come sufficiently close that they cannot be distinguished as separate objects at the resolution of the light microscope (within 200nm) for these connections to occur (Corson and Erisir 2013). Therefore, the largest number of taste-transducing cells that are sufficiently close to a neuron to form a connection can be determined. To determine the closest proximity between taste-transducing cells and nerve fibers, we labeled all taste nerve arbors (Phox2b-Cre:tdTomato mice) and many taste-transducing cells using well-established markers for cells transducing sweet, bitter, and umami-type stimuli [(anti-phospholipase C beta-2 (PLCß2) (Zhao, Zhang et al. 2003, Clapp, Yang et al. 2004)] and those transducing sour-stimuli [(anti-carbonic anhydrase 4 (Car4) (Chandrashekar, Yarmolinsky et al. 2009)]. We then analyzed three-dimensional (3D) image stacks to identify the taste-transducing cells and nerve arbors that were closest to each other (Fig. 8A). We found that nerve arbors tended to be either within one voxel $(\sim 110 \mathrm{~nm}$, below the resolution of the light microscope and frequently seen as overlapping (referred to henceforth as contacts) or separated by more than $1 \mu \mathrm{m}$ (Fig. 8A,B). The micron 
separation between non-overlapping nerve fibers and labeled taste bud cells is likely due to the unlabeled supporting cells that comprise the bulk of the taste bud (Yang, Dzowo et al. 2019), which have processes that wrap around nerve arbors and taste-transducing cells (Bartel, Sullivan et al. 2006). While individual contacts may not be particularly meaningful, numbers or patterns can be compared across groups. For example, taste neurons expressing the serotonin receptor contact more sour-transducing cells (which produce serotonin) than other cell types (Stratford, Larson et al. 2017).

To determine how many taste-transducing cells are contacted by individual taste arbors, we reconstructed 151 individual arbors in fungiform taste buds. Arbors contacting either PLCß2labeled (Fig. 8C,D) or Car4-labeled taste bud cells (Fig. 8E,F) sometimes followed the labeled

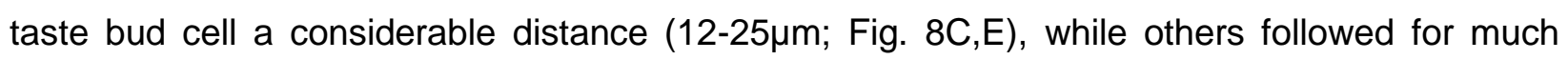
shorter distances (less than $12 \mu \mathrm{m}$, Fig. 8D,F). Frequently, arbors contact the same cell at multiple different locations. Because it is unclear how many of these contacts actually represent a connection, we did not quantify contacts, but quantified the number of cells contacted. Thus, an arbor contacts one cell regardless of the distance travelled along the cell or the number of distinct instances in which the nerve arbor or taste receptor cell come within $200 \mathrm{~nm}$. Using this analysis, we found that each arbor contacted 1.87 taste-transducing cells on average. This number is only slightly more than the average of 1.6 Type III cells that synapse with individual circumvallate arbors (Kinnamon, Sherman et al. 1988).

We found that $48 \%$ of the arbors only contacted one taste-transducing cell, suggesting that these arbors either form a connection with a single taste-transducing cell or do not form a connection at all. A few of the arbors contacted no labeled taste bud cells (7\%) and could not receive input from either a Car4-positive or PLCß2-positive taste bud cell. Of those that contacted multiple cells, 26 contacted cells of the same type (17\%; only Car4- or only PLCß2-positive cells) and 43 contacted both types (28\%; PLCß2- and Car4-labeled taste bud cells). In addition to 
functional connections, an arbor likely contacts a taste-transducing cell when assessing molecular compatibility (Lee, Macpherson et al. 2017) to form a new connection or when an branch passes in close proximity to a receptor cell. Because there is no published evidence that an individual arbor can receive functional input from more than one transducing cell type, we hypothesized that arbors contacting more than one cell type might be larger, and perhaps in a state of greater plasticity (process of connecting to a new cell). Consistent with this possibility, arbors contacting more than one cell type are longer (median=89 $(\mathrm{N}=106)$ vs $57(\mathrm{~N}=39) \mu \mathrm{m}, \mathrm{U}=1185, p=0.0001)$ and more heavily branched (median $5(\mathrm{~N}=106)$ vs 3 branch ends $(\mathrm{N}=39), \mathrm{U}=1522, p=0.0012)$.

\section{Complex neurons contact more taste-transducing cells than simply branched neurons.}

Next, we sought to examine the degree to which more heavily branched neurons have an opportunity to connect with larger numbers of taste-transducing cells. To this end, we labeled only one axon entering each half of the tongue, so that all the individual arbors identified were from the same neuron. Since these neurons typically do not cross the midline, each half of the tongue can be evaluated independently. Individual axons were identified from where the chorda tympanilingual nerve enters the tongue in a single nerve bundle and followed through multiple serial sections to determine the number of labeled axons (Fig. 9A). By injecting different low doses of tamoxifen $(0.3-0.6 \mathrm{mg})$, we determined that one injection of $0.6 \mathrm{mg}$ results in one single labeled axon roughly $25 \%$ of the time. For each axon verified as a single labeled axon in one tongue half, all 40-45 taste buds on that side of the tongue were immunolabeled and scanned by confocal microscopy to identify and reconstruct each terminal arbor. Three examples of cluster 1 neurons are illustrated (Figure 9B). The first neuron innervated one taste bud and contacted two Car4positive cells. The second neuron innervated two taste buds and contacted 4 PLCß2-positive cells, and the third neuron contacts one PLCß2-postive cell. A single cluster 2 neuron is also illustrated (Fig. 9C), which contacted 10 labeled taste cells across 5 taste buds. Although this axon contacts 
multiple taste cell types, the contacts were heavily biased toward Car4-positive cells (7 Car4 and 3 PLCß2).

A total of 21 single axons from clusters 1, 2, and 3 were analyzed. We found that the mean number of taste-transducing cells contacted significantly differed between clusters $\left(H_{(2,20)}=17.16\right.$ $p=0.0002$; Kruskal-Wallis; Fig. 9D). In fact, most cluster 1 neurons only contact one PLCß2labeled taste cell. This finding indicates that either many taste neurons are either unresponsive to stimuli or a neuron response can be driven by input from only a single taste-transducing cell. Another difference across these morphological clusters was the pattern in the type of tastetransducing cells contacted across the clusters. Arbors from the cluster 1 neurons are more likely to contact a PLCß2 cell and less likely to contact a Car4 cell than terminal arbors from clusters 2 and 3 neurons (Fig.9E, $x^{2}=6.63, p=0.036$ ).

We examined this same question another way using a similar approach as functional studies. Functionally, gustatory neurons are typically divided based on their "best" stimulus quality (sweet, sour, etc.), even though roughly $40 \%$ of the population responds to more than one stimulus (Frank 2000, Yoshida, Yasumatsu et al. 2006, Barretto, Gillis-Smith et al. 2015, Wu, Dvoryanchikov et al. 2015). We speculated that a similar approach would permit us to compare morphologies across predicted functional quality by dividing neurons based on the type of cell contacted most frequently, PLC $\beta 2$ (sweet/bitter) or Car4 (sour) cells. For example, the neuron in Figure 9C contacted 7 Car4-positive cells and 3 PLCß2-positive cells, so it was placed in the group contacting more Car4 cells. The two neurons that contact the same number of each cell type were placed into groups based on the combined size of these contacts. Of the 21 neurons examined, 11 contacted more sweet/bitter cells, while 10 contacted more sour-transducing cells. Neurons contacting more sour-transducing cells had a greater combined terminal arbor length (Fig.9F;t $\left.\mathrm{t}_{(19)} 2.59, \mathrm{p}=0.018\right)$ and more branch ends (median $=5(\mathrm{~N}=11)$ vs $\left.16(\mathrm{~N}=10) \mathrm{U}=19, \mathrm{p}=0.025\right)$, indicating that they are more heavily branched than neurons primarily contacting 
bioRxiv preprint doi: https://doi.org/10.1101/2020.08.20.260059; this version posted December 14, 2020. The copyright holder for this preprint (which was not certified by peer review) is the author/funder. All rights reserved. No reuse allowed without permission.

sweet/bitter/umami-transducing cells. However, there was also considerable variation within each group and distributions were overlapping (Fig. 9F). 


\section{Discussion}

Taste neurons exhibit both functional (Frank 1973, Lundy and Contreras 1999, Frank 2000, Sollars and Hill 2005, Yoshida, Yasumatsu et al. 2006, Breza, Nikonov et al. 2010, Yoshida and Ninomiya 2010, Barretto, Gillis-Smith et al. 2015, Wu, Dvoryanchikov et al. 2015) and molecular diversity (Yee, Yang et al. 2001, Zhang, Jin et al. 2019). Our goal was to determine if they are also morphologically diverse. Using sparse-cell genetic labeling, we found considerable variation in the branching characteristics of taste neurons. Roughly half of the taste neurons had few branches (cluster 1), whereas others branched extensively along a continuum of complexity. Axons of cluster 2 neurons had more arbors and innervated more taste buds than cluster 1 neurons (maximum 7 taste buds). Whereas the more heavily branched axons of cluster 3 and 4 neurons innervated a similar number of taste buds as cluster 2 neurons, but had more terminal arbors per taste bud (maximum 17 arbors). This variability in branching complexity likely introduces variation in the amount of convergence of taste-transducing cell input onto individual neurons. We were able to determine the largest possible number of potential connections for 21 neurons. We found that simple neurons (cluster 1 ) typically only contacted 1-4 taste-transducing cells, whereas more heavily branched cluster 2 and 3 neurons could potentially receive input from 5-22 taste-transducing cells. In addition, more heavily branched neurons were more likely to contact sour-transducing cells than those that transduce sweet/better, indicating that neuron types (divided based on taste quality) likely differ in morphology. Lastly, variation in morphology within a neuron type or quality is also likely and has important implications for both function and plasticity.

To what extent the variable morphological complexities represent a snap-shot in time of a changing pattern or are permanent characteristics is unclear. A unique feature of taste bud cells is that they have a limited lifespan and are constantly renewed (Beidler and Smallman 1965, Farbman 1980, Delay, Kinnamon et al. 1986, Perea-Martinez, Nagai et al. 2013). As a result, gustatory ganglion neurons must continually locate and form functional connections with new adult taste-transducing cells. Presumably, this process is accompanied by changes in branching 
characteristics. We observed substantial variation in neuron complexity which predicts differences in the number of taste-transducing cells contacted by individual neurons. If branching changes over time, degree of convergence could also be a changing feature of taste neurons resulting in changing functional characteristics over time (Shimatani, Nikles et al. 2003). However, this may be true only within a limited range if some components of the neuron structure are stable over time.

One possibility is that arbor complexity is dictated primarily by the process of finding and connecting with a new taste-transducing cell, while arbor number is a stable feature of the neuron. We found no differences in mean terminal arbor length or complexity across clusters, consistent with the idea that terminal arbor structure is primarily plastic. The largest/most complex terminal arbors could be extending throughout the taste bud to locate and connect to a new tastetransducing cell. Consistently, terminal arbors contacting multiple cell types were longer and more heavily branched. In spite of their variable morphology, most terminal arbors only contact 1-2 taste-transducing cells, which is consistent with electron microscopy findings for synapses (Kinnamon, Sherman et al. 1988). Consistently, we found that the number of terminal arbors is the best predictor of the number of taste-transducing cells contacted by an individual neuron. The number of terminal arbors is determined by the number of branch points below the epithelium. Because a change in these branch points may not be required for a taste bud to form a new connection with a taste-transducing cell, the number of arbors may be a stable characteristic of the neuron. Because number of arbors determines the number of taste-transducing cells contacted this could contribute to functional stability over time.

We examined the number of taste-transducing cells contacted by each neuron. Contacts likely represent one of three scenarios, only one of which represents functional connectivity. First, there are likely some locations where the nerve arbor simply passes within $200 \mathrm{~nm}$ of a tastetransducing cell without any specific interaction with that cell. A second possibility are locations where the cell membranes of neurons and taste-transducing cells contact as part of the process 
of re-innervation with cell turnover (as discussed above). Contact between taste-transducing cells and nerve arbors would allow a nerve arbor to determine molecular compatibility (i.e. the presence of a ligand on one cell and receptor on the other for factors involved in synapse formation). These "sampling contacts" are likely since the continued rewiring of the taste system is thought to depend upon multiple molecular factors (Lee, Macpherson et al. 2017). The third scenario is that all functional connections require a contact measurable at the light level. Distinguishing between these possibilities for an individual neuron is not possible even with EM analysis (Yang, Dzowo et al. 2019). This is the case because individual arbors from the same neuron, but innervating different taste buds, are separated by too great a distance to be reconstructed at the EM level. In addition, structural correlates do not exist between nerve fibers and all cell types, in spite of recent advances (Romanov, Lasher et al. 2018). However, it seems likely that the number of functional connections is greater in neurons with many arbors than for neurons with only a single arbor. If each neuron only had a functional connection on only one terminal arbor, regardless of the number terminal arbors, only 96 of the 452 arbors we observed would have a connection, leaving $78 \%$ of all arbors without a connection to a taste-transducing cell - a possibility which is not consistent with EM studies (Kinnamon, Sherman et al. 1988). Thus, the most likely reason for differential branching is differential convergence.

Variations in the amount of convergent input from the same type of taste-transducing onto different neurons could result in variable sensitivities to the taste stimulus transduced by these cells. For example, a neuron receiving input from eight sour-transducing cells (Car4 expressing (Huang, Chen et al. 2006, Chandrashekar, Yarmolinsky et al. 2009)) may be more sensitive to citric acid than a neuron receiving input from only two sour transducing cells. Consistent with this idea, stimulation of independent areas of a neuron's receptive field with the same stimulus enhances the response (Miller 1971). Our results show that not all neurons branch to contact multiple taste-transducing cells, suggesting that this enhancement occurs in some neurons, but not others. These differences could produce variations in thresholds and intensity ranges for the 
same stimulus across the population of neurons. While increases in response rate represent taste stimulus intensity (Ganchrow and Erickson 1970, Scott, Plata-Salaman et al. 1991, Breza, Nikonov et al. 2010, Fonseca, de Lafuente et al. 2018), it is unclear whether additional peripheral neurons are recruited as stimulus intensity increases (i.e. graded intensity coding), as is the case with warm stimuli (Wang, Belanger et al. 2018). Variation in stimulus thresholds across the population is consistent with our anatomical data and would permit a greater range of intensities to be coded by taste neurons than by individual taste-transducing cells (Caicedo, Kim et al. 2002). This possibility is supported by the findings from the few studies that utilized multiple stimulus concentrations to examine taste coding in peripheral neurons (Ganchrow and Erickson 1970, Breza, Nikonov et al. 2010, Wu, Dvoryanchikov et al. 2015). Consistent with this idea, our data predict a range of branching characteristics within each taste quality.

We also observed that neurons contacting more sour-transducing cells tended to be more heavily branched than those contacting sweet/bitter/umami transducing cells. If as a result, neurons responding to sour stimuli receive input from a larger number of taste-transducing cells, they would be predicted to be more broadly tuned. Approximately one-fourth of individual tastetransducing cells are capable of responding to more than one stimulus (Tomchik, Berg et al. 2007, Yoshida and Ninomiya 2016). Therefore, as the amount of convergent input increases for a given neuron, the probability that it will receive input from taste-transducing cells responding to multiple stimuli also increases. Variation in branching may explain why functional studies of peripheral taste neurons have consistently observed both narrowly and broadly tuned neurons (Yoshida, Yasumatsu et al. 2006, Barretto, Gillis-Smith et al. 2015, Wu, Dvoryanchikov et al. 2015). Consistently, neurons responding to sweet are typically described as more narrowly tuned than neurons responding to primarily to sour (Lundy and Contreras 1999, Frank 2000, Breza, Nikonov et al. 2010). While it has been repeatedly speculated that this is due to differences in the tuning properties of the taste-transducing cells (Tomchik, Berg et al. 2007, Barretto, Gillis-Smith et al. 
bioRxiv preprint doi: https://doi.org/10.1101/2020.08.20.260059; this version posted December 14, 2020. The copyright holder for this preprint (which was not certified by peer review) is the author/funder. All rights reserved. No reuse allowed without permission.

2015), our data suggest that differential convergence onto the nerve fiber is another likely explanation. 


\section{References} 639.

Abraira, V. E. and D. D. Ginty (2013). "The sensory neurons of touch." Neuron 79(4): 618-

Bachmanov, A. A., M. G. Tordoff and G. K. Beauchamp (1996). "Ethanol consumption and taste preferences in C57BL/6ByJ and 129/J mice." Alcohol Clin Exp Res 20(2): 201-206.

Badea, T. C., Y. Wang and J. Nathans (2003). "A noninvasive genetic/pharmacologic strategy for visualizing cell morphology and clonal relationships in the mouse." J Neurosci 23(6): 2314-2322.

Badea, T. C., J. Williams, P. Smallwood, M. Shi, O. Motajo and J. Nathans (2012). "Combinatorial expression of Brn3 transcription factors in somatosensory neurons: genetic and morphologic analysis." J Neurosci 32(3): 995-1007.

Bai, L., B. P. Lehnert, J. Liu, N. L. Neubarth, T. L. Dickendesher, P. H. Nwe, C. Cassidy, C. J. Woodbury and D. D. Ginty (2015). "Genetic Identification of an Expansive Mechanoreceptor Sensitive to Skin Stroking." Cell 163(7): 1783-1795.

Barretto, R. P., S. Gillis-Smith, J. Chandrashekar, D. A. Yarmolinsky, M. J. Schnitzer, N. J. Ryba and C. S. Zuker (2015). "The neural representation of taste quality at the periphery." Nature 517(7534): 373-376.

Bartel, D. L., S. L. Sullivan, E. G. Lavoie, J. Sevigny and T. E. Finger (2006). "Nucleoside triphosphate diphosphohydrolase-2 is the ecto-ATPase of type I cells in taste buds." J Comp Neurol 497(1): 1-12.

Beidler, L. M. and R. L. Smallman (1965). "Renewal of cells within taste buds." J Cell Biol 27(2): 263-272.

Bernstein, M. and J. W. Lichtman (1999). "Axonal atrophy: the retraction reaction." $\underline{\text { Curr }}$ Opin Neurobiol 9(3): 364-370.

Breza, J. M., K. S. Curtis and R. J. Contreras (2006). "Temperature modulates taste responsiveness and stimulates gustatory neurons in the rat geniculate ganglion." $\mathrm{J}$ Neurophysiol 95(2): 674-685.

Breza, J. M., A. A. Nikonov and R. J. Contreras (2010). "Response latency to lingual taste stimulation distinguishes neuron types within the geniculate ganglion." J Neurophysiol 103(4): 1771-1784.

Caicedo, A., K. N. Kim and S. D. Roper (2002). "Individual mouse taste cells respond to multiple chemical stimuli." J Physiol 544(2): 501-509.

Carleton, A., R. Accolla and S. A. Simon (2010). "Coding in the mammalian gustatory system." Trends Neurosci 33(7): 326-334.

Castillo, K., I. Diaz-Franulic, J. Canan, F. Gonzalez-Nilo and R. Latorre (2018). "Thermally activated TRP channels: molecular sensors for temperature detection." Phys Biol 15(2): 021001.

Chandrashekar, J., M. A. Hoon, N. J. Ryba and C. S. Zuker (2006). "The receptors and cells for mammalian taste." Nature 444(7117): 288-294.

Chandrashekar, J., D. Yarmolinsky, L. von Buchholtz, Y. Oka, W. Sly, N. J. Ryba and C. S. Zuker (2009). "The taste of carbonation." Science 326(5951): 443-445.

Chklovskii, D. B. (2004). "Synaptic connectivity and neuronal morphology: two sides of the same coin." Neuron 43(5): 609-617.

Clapp, T. R., R. Yang, C. L. Stoick, S. C. Kinnamon and J. C. Kinnamon (2004). "Morphologic characterization of rat taste receptor cells that express components of the phospholipase C signaling pathway." J Comp Neurol 468(3): 311-321.

Contreras, R. J. and R. F. Lundy (2000). "Gustatory neuron types in the periphery: a functional perspective." Physiol Behav 69(1-2): 41-52.

Corson, J. A. and A. Erisir (2013). "Monosynaptic convergence of chorda tympani and glossopharyngeal afferents onto ascending relay neurons in the nucleus of the solitary tract: a 
high-resolution confocal and correlative electron microscopy approach." J Comp Neurol 521(13): 2907-2926.

Delay, R. J., J. C. Kinnamon and S. D. Roper (1986). "Ultrastructure of mouse vallate taste buds: II. Cell types and cell lineage." J Comp Neurol 253(2): 242-252.

Dvoryanchikov, G., D. Hernandez, J. K. Roebber, D. L. Hill, S. D. Roper and N. Chaudhari (2017). "Transcriptomes and neurotransmitter profiles of classes of gustatory and somatosensory neurons in the geniculate ganglion." Nat Commun 8(1): 760.

Farbman, A. I. (1980). "Renewal of taste bud cells in rat circumvallate papillae." Cell Tissue Kinet 13(4): 349-357.

Fonseca, E., V. de Lafuente, S. A. Simon and R. Gutierrez (2018). "Sucrose intensity coding and decision-making in rat gustatory cortices." Elife 7.

Frank, M. (1973). "An analysis of hamster afferent taste nerve response functions." J Gen Physiol 61(5): 588-618.

Frank, M. E. (2000). "Neuron types, receptors, behavior, and taste quality." Physiol Behav 69(1-2): 53-62.

Ganchrow, J. R. and R. P. Erickson (1970). "Neural correlates of gustatory intensity and quality." J Neurophysiol 33(6): 768-783.

Guagliardo, N. A. and D. L. Hill (2007). "Fungiform taste bud degeneration in C57BL/6J mice following chorda-lingual nerve transection." J Comp Neurol 504(2): 206-216.

Huang, A. L., X. Chen, M. A. Hoon, J. Chandrashekar, W. Guo, D. Trankner, N. J. Ryba and C. S. Zuker (2006). "The cells and logic for mammalian sour taste detection." Nature 442(7105): 934-938.

Huang, T. and R. F. Krimm (2010). "Developmental expression of Bdnf, Ntf4/5, and TrkB in the mouse peripheral taste system." Dev Dyn 239(10): 2637-2646.

Kinnamon, J. C., T. A. Sherman and S. D. Roper (1988). "Ultrastructure of mouse vallate taste buds: III. Patterns of synaptic connectivity." J Comp Neurol 270(1): 1-10, 56-17.

Kuehn, E. D., S. Meltzer, V. E. Abraira, C. Y. Ho and D. D. Ginty (2019). "Tiling and somatotopic alignment of mammalian low-threshold mechanoreceptors." Proc Natl Acad Sci U S A 116(19): 9168-9177.

Lee, H., L. J. Macpherson, C. A. Parada, C. S. Zuker and N. J. P. Ryba (2017). "Rewiring the taste system." Nature 548(7667): 330-333.

Liebl, D. J., J. P. Mbiene and L. F. Parada (1999). "NT4/5 mutant mice have deficiency in gustatory papillae and taste bud formation." Dev Biol 213(2): 378-389.

Lim, J. and A. J. Pullicin (2019). "Oral carbohydrate sensing: Beyond sweet taste." Physiol Behav 202: 14-25.

Liu, D., N. Archer, K. Duesing, G. Hannan and R. Keast (2016). "Mechanism of fat taste perception: Association with diet and obesity." Prog Lipid Res 63: 41-49.

Lundy, R. F., Jr. and R. J. Contreras (1997). "Temperature and amiloride alter taste nerve responses to $\mathrm{Na}+, \mathrm{K}+$, and $\mathrm{NH}+4$ salts in rats." Brain Res 744(2): 309-317.

Lundy, R. F., Jr. and R. J. Contreras (1999). "Gustatory neuron types in rat geniculate ganglion." J Neurophysiol 82(6): 2970-2988.

Ma, Z., A. Taruno, M. Ohmoto, M. Jyotaki, J. C. Lim, H. Miyazaki, N. Niisato, Y. Marunaka, R. J. Lee, H. Hoff, R. Payne, A. Demuro, I. Parker, C. H. Mitchell, J. Henao-Mejia, J. E. Tanis, I. Matsumoto, M. G. Tordoff and J. K. Foskett (2018). "CALHM3 Is Essential for Rapid lon ChannelMediated Purinergic Neurotransmission of GPCR-Mediated Tastes." Neuron 98(3): 547-561 e510.

Maksimovic, S., M. Nakatani, Y. Baba, A. M. Nelson, K. L. Marshall, S. A. Wellnitz, P. Firozi, S. H. Woo, S. Ranade, A. Patapoutian and E. A. Lumpkin (2014). "Epidermal Merkel cells are mechanosensory cells that tune mammalian touch receptors." Nature 509(7502): 617-621.

Miller, I. J., Jr. (1971). "Peripheral interactions among single papilla inputs to gustatory nerve fibers." J Gen Physiol 57(1): 1-25. 
Murray, R. G. (1986). "The mammalian taste bud type III cell: a critical analysis." J Ultrastruct Mol Struct Res 95(1-3): 175-188.

Ohla, K., R. Yoshida, S. D. Roper, P. M. Di Lorenzo, J. D. Victor, J. D. Boughter, M. Fletcher, D. B. Katz and N. Chaudhari (2019). "Recognizing taste: coding patterns along the neural axis in mammals." Chem Senses.

Ohman-Gault, L., T. Huang and R. Krimm (2017). "The transcription factor Phox2b distinguishes between oral and non-oral sensory neurons in the geniculate ganglion." J Comp Neurol 525(18): 3935-3950.

Patel, A. V. and R. F. Krimm (2012). "Neurotrophin-4 regulates the survival of gustatory neurons earlier in development using a different mechanism than brain-derived neurotrophic factor." Dev Biol 365(1): 50-60.

Perea-Martinez, I., T. Nagai and N. Chaudhari (2013). "Functional cell types in taste buds have distinct longevities." PLoS One 8(1): e53399.

Rios-Pilier, J. and R. F. Krimm (2019). "TrkB expression and dependence divides gustatory neurons into three subpopulations." Neural Dev 14(1): 3.

Romanov, R. A., R. S. Lasher, B. High, L. E. Savidge, A. Lawson, O. A. Rogachevskaja, H. Zhao, V. V. Rogachevsky, M. F. Bystrova, G. D. Churbanov, I. Adameyko, T. Harkany, R. Yang, G. J. Kidd, P. Marambaud, J. C. Kinnamon, S. S. Kolesnikov and T. E. Finger (2018). "Chemical synapses without synaptic vesicles: Purinergic neurotransmission through a CALHM1 channelmitochondrial signaling complex." Sci Signal 11(529).

Rutlin, M., C. Y. Ho, V. E. Abraira, C. Cassidy, C. J. Woodbury and D. D. Ginty (2014). "The cellular and molecular basis of direction selectivity of Adelta-LTMRs." Cell 159(7): 1640-1651.

Scott, T. R., C. R. Plata-Salaman, V. L. Smith and B. K. Giza (1991). "Gustatory neural coding in the monkey cortex: stimulus intensity." J Neurophysiol 65(1): 76-86.

Shimatani, Y., S. A. Nikles, K. Najafi and R. M. Bradley (2003). "Long-term recordings from afferent taste fibers." Physiol Behav 80(2-3): 309-315.

Sollars, S. I. and D. L. Hill (2005). "In vivo recordings from rat geniculate ganglia: taste response properties of individual greater superficial petrosal and chorda tympani neurones." $\underline{\mathrm{J}}$ Physiol 564(Pt 3): 877-893.

Spector, A. C. and S. P. Travers (2005). "The representation of taste quality in the mammalian nervous system." Behav Cogn Neurosci Rev 4(3): 143-191.

Stratford, J. M., E. D. Larson, R. Yang, E. Salcedo and T. E. Finger (2017). "5-HT3A -driven green fluorescent protein delineates gustatory fibers innervating sour-responsive taste cells: A labeled line for sour taste?" J Comp Neurol 525(10): 2358-2375.

Sun, C., A. Dayal and D. L. Hill (2015). "Expanded terminal fields of gustatory nerves accompany embryonic BDNF overexpression in mouse oral epithelia." J Neurosci 35(1): 409-421.

Tang, T., J. Rios-Pilier and R. Krimm (2017). "Taste bud-derived BDNF maintains innervation of a subset of TrkB-expressing gustatory nerve fibers." Mol Cell Neurosci 82: 195-203.

Taruno, A., V. Vingtdeux, M. Ohmoto, Z. Ma, G. Dvoryanchikov, A. Li, L. Adrien, H. Zhao, S. Leung, M. Abernethy, J. Koppel, P. Davies, M. M. Civan, N. Chaudhari, I. Matsumoto, G. Hellekant, M. G. Tordoff, P. Marambaud and J. K. Foskett (2013). "CALHM1 ion channel mediates purinergic neurotransmission of sweet, bitter and umami tastes." Nature 495(7440): 223-226.

Tomchik, S. M., S. Berg, J. W. Kim, N. Chaudhari and S. D. Roper (2007). "Breadth of tuning and taste coding in mammalian taste buds." J Neurosci 27(40): 10840-10848.

Tordoff, M. G. (2001). "Calcium: taste, intake, and appetite." Physiol Rev 81(4): 1567-1597. 425-433.

Tordoff, M. G. (2017). "Phosphorus Taste Involves T1R2 and T1R3." Chem Senses 42(5):

Valm, A. M., S. Cohen, W. R. Legant, J. Melunis, U. Hershberg, E. Wait, A. R. Cohen, M. W. Davidson, E. Betzig and J. Lippincott-Schwartz (2017). "Applying systems-level spectral imaging and analysis to reveal the organelle interactome." Nature 546(7656): 162-167. 
Wang, F., E. Belanger, S. L. Cote, P. Desrosiers, S. A. Prescott, D. C. Cote and Y. De Koninck (2018). "Sensory Afferents Use Different Coding Strategies for Heat and Cold." Cell Rep 23(7): 2001-2013.

Wu, A., G. Dvoryanchikov, E. Pereira, N. Chaudhari and S. D. Roper (2015). "Breadth of tuning in taste afferent neurons varies with stimulus strength." Nat Commun 6: 8171.

Wu, H., J. Williams and J. Nathans (2012). "Morphologic diversity of cutaneous sensory afferents revealed by genetically directed sparse labeling." Elife 1: e00181.

Wu, P., D. Arris, M. Grayson, C. N. Hung and S. Ruparel (2018). "Characterization of sensory neuronal subtypes innervating mouse tongue." PLoS One 13(11): e0207069.

Yang, R., Y. K. Dzowo, C. E. Wilson, R. L. Russell, G. J. Kidd, E. Salcedo, R. S. Lasher, J. C. Kinnamon and T. E. Finger (2019). "Three-dimensional reconstructions of mouse circumvallate taste buds using serial blockface scanning electron microscopy: I. Cell types and the apical region of the taste bud." J Comp Neurol.

Yarmolinsky, D. A., C. S. Zuker and N. J. Ryba (2009). "Common sense about taste: from mammals to insects." Cell 139(2): 234-244.

Yee, C. L., R. Yang, B. Bottger, T. E. Finger and J. C. Kinnamon (2001). "'Type III" cells of rat taste buds: immunohistochemical and ultrastructural studies of neuron-specific enolase, protein gene product 9.5, and serotonin." J Comp Neurol 440(1): 97-108.

Yokota, Y. and R. M. Bradley (2016). "Receptive field size, chemical and thermal responses, and fiber conduction velocity of rat chorda tympani geniculate ganglion neurons." $\mathrm{J}$ Neurophysiol 115(6): 3062-3072.

Yokota, Y. and R. M. Bradley (2017). "Geniculate Ganglion Neurons are Multimodal and Variable in Receptive Field Characteristics." Neuroscience 367: 147-158.

Yoshida, R. and Y. Ninomiya (2010). "New insights into the signal transmission from taste cells to gustatory nerve fibers." Int Rev Cell Mol Biol 279: 101-134.

Yoshida, R. and Y. Ninomiya (2016). "Taste information derived from T1R-expressing taste cells in mice." Biochem J 473(5): 525-536.

Yoshida, R., K. Yasumatsu, N. Shigemura and Y. Ninomiya (2006). "Coding channels for taste perception: information transmission from taste cells to gustatory nerve fibers." Arch Histol Cytol 69(4): 233-242.

Zaidi, F. N., V. Cicchini, D. Kaufman, E. Ko, A. Ko, H. Van Tassel and M. C. Whitehead (2016). "Innervation of taste buds revealed with Brainbow-labeling in mouse." J Anat 229(6): 778790.

Zaidi, F. N., R. F. Krimm and M. C. Whitehead (2007). "Exuberant neuronal convergence onto reduced taste bud targets with preservation of neural specificity in mice overexpressing neurotrophin in the tongue epithelium." J Neurosci 27(50): 13875-13881.

Zaidi, F. N. and M. C. Whitehead (2006). "Discrete innervation of murine taste buds by peripheral taste neurons." J Neurosci 26(32): 8243-8253.

Zhang, J., H. Jin, W. Zhang, C. Ding, S. O'Keeffe, M. Ye and C. S. Zuker (2019). "Sour Sensing from the Tongue to the Brain." Cell 179(2): 392-402 e315.

Zhao, G. Q., Y. Zhang, M. A. Hoon, J. Chandrashekar, I. Erlenbach, N. J. Ryba and C. S. Zuker (2003). "The receptors for mammalian sweet and umami taste." Cell 115(3): 255-266. 


\section{Figure legends}

Figure 1. Genetically directed sparse-cell labeling, reconstruction, and quantification of individual

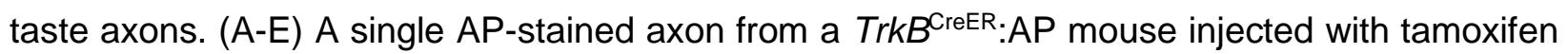
at postnatal day 40. (A) A reconstruction of the taste axon starting from where it enters the tongue (B) to the terminal arbors. It is superimposed on an outline of a tongue section. (B) Images of the AP-stained axon are shown at 3 different focal depths (left to right) in this 180- $\mu$ m section. (C) An enlarged view of the same axon with each branch segment presented in a different color. This axon innervates 4 taste buds (one of which is shown in $\mathrm{D}$ ). Boxes illustrate the locations of branch points (E'-E'), which are each shown at 3 different focal depths. (F) illustrates 7 of the features quantified for the entire axon (in the legend), using the axon shown (A-E) as an example. In addition, the total length of the axon was measured (green and gray in F summed, 12.7mm). The location in the tongue was assigned a number (tip=1, mid-region=2, back=3); for the illustrated neuron 1.5 was assigned, since it had arbors innervated both the tip and mid-region. (G-H) Illustrate 7 additional anatomical characteristics measured in the taste buds. The scale bar in $\mathrm{B}=$ $20 \mu \mathrm{m}$ and applies to $\mathrm{D}$ and $\mathrm{E}$; the scale bar in $\mathrm{C}=100 \mu \mathrm{m}$.

Figure 2. Anatomic receptive fields tend to be distributed over the lingual epithelial surface. (A) An example of an axon that innervated 5 taste buds, with a total of 6 terminal arbors (only one of these taste buds received more than one arbor). The fungiform papillae containing the innervated taste buds are marked by black dots. Other fungiform papillae containing taste buds not innervated by this neuron (marked by gray dots) and at least two are located between innervated papillae. Approximately $10 \%$ of axons have these extensive receptive fields. (B) A histogram illustrating the number of taste buds innervated by 1 to 6 terminal arbors from the same neuron. The 96 reconstructed axons innervate a total of 279 taste buds, and most taste buds only receive a single arbor from each taste neuron.

Figure 3. Most, but not all, TrkB-positive taste axons projecting to the tongue innervate taste buds. (A) On the un-transected side of the tongue (intact), abundant TrkB-positive axons (magenta) were observed in chorda-lingual nerve bundles. (B) On the chorda tympani transected side of the tongue (CTX) from the same mouse, substantially fewer TrkB-positive axons were observed in nerve bundles. (C) TrkB-positive axon terminals were clearly seen in taste buds labeled by cytokeratin-8 (enclosed with dashed line), from the intact side of the tongue (indicated by arrows in E). With high doses of tamoxifen label is also present in most of the non-taste epithelia around the taste bud $(C-F)$. (D,F) On the CTX side of the tongue, taste buds lacked labeled axons. Scale bar in $B=50 \mu \mathrm{m}$ and also applies to A; scale bar in $F=10 \mu \mathrm{m}$ and also applies to $\mathrm{C}, \mathrm{D}$, and $\mathrm{E}$. 
Figure 4. Taste axons were divided into four categories using a k-means cluster analysis. (A) Silhouette values are graphed for each neuron based on the similarity of each neuron to its own cluster (higher values = greater similarity). Four plots illustrate the number of clusters varying from 2 to 5 . The highest silhouette value (0.72) was obtained assuming there were only two clusters, but the two-cluster model showed outliers. which also observed in the cluster 3 and the cluster 5 models. A four cluster model had the second highest mean silhouette value $(0.69)$ with no outliers. (B-D) The distributions of combined terminal arbor length, total number of branch ends, highest branch order. These are the measures showing the greatest separation between clusters. (E) 3D graph showing the relationship of these three characteristics for individual neurons. $(F)$ Clusters are defined by combined arbor length, which is the amount of the axon available to form a connection with a taste receptor cells. ${ }^{* \star} p \leq 0.001$

Figure 5. Two examples of axons from cluster 1 neurons. (A, B) The reconstructed axons were superimposed on an outline of one sagittal tongue section to illustrate the location of the axon in the tongue. The images below each reconstructed axon show the AP staining of terminal arbors within the taste bud at three different focal depths. Reconstructions of the terminal arbors within the taste bud are shown at the same magnification to the right of the images. (A) This 10.3-mmlong axon innervated a single taste bud with one terminal arbor, three branch points inside the taste bud, and four branch ends (gray arrowheads). (B) The second 14.0-mm-long axon innervated two taste buds with one terminal arbor each. One branch point outside the taste bud and four inside the taste bud, results in six total branch ends (gray arrowheads). (C) Axons from cluster 1 taste neurons innervate 1-4 taste buds. The number of taste buds that are innervated correlates with the total length of the axon. (D) The axons of cluster 1 neurons typically have 1 to 2 terminal arbors penetrating taste buds, but could have as many as 6 terminal arbors. (E) Axons of cluster 1 neurons with the most terminal arbors also had on average the shortest terminal arbors. (F) The distribution of the number of branch endings per terminal arbor. Most axons from cluster 1 neurons had terminal arbors with 2 branch ends. Scale bars: $1 \mathrm{~mm}$ for whole axon tracings in $A$ and $B ; 10 \mu m$ for taste bud images and reconstructions within taste buds in $A$ and $B$.

Figure 6. Two examples of axons from cluster 2 neurons. The reconstructed axons were superimposed on an outline of one sagittal tongue section to illustrate the location of the axon in the tongue. The images below each reconstructed axon show the AP staining of terminal arbors within the taste bud at three different focal depths. Reconstructions of the terminal arbors within the taste bud are shown at the same magnification to the right of the images. (A) The $13.0 \mathrm{~mm}$ axon of this cluster 2 neuron had 3 branch points below the epithelium producing 4 terminal arbors 
that innervated 3 taste buds (second arbor in same taste bud is green). Inside the taste bud some of the arbors had additional branch points (5) to produce 10 branch ends (gray arrowheads). The imaged taste bud has a single arbor (blue arrow) from the reconstructed axon plus one arbor from another labeled neuron (red arrow). Beneath the images are reconstructions of 3 additional terminal arbors innervating two additional taste buds. (B) The $14.7 \mathrm{~mm}$ axon from a second neuron had 6 branch points below the epithelia, producing 7 terminal arbors, each of which innervated a separate taste bud (reconstructions for 6 additional arbors are shown beneath the imaged arbor). Some of these arbors also branched in the taste bud (6 branch points) resulting in a total of 13 terminal branch ends (gray arrowheads). (C) Plots of medians \pm interquartile ranges (IQR, gray boxes) and \pm maximum/minimum (whiskers) illustrate that cluster 2 neurons innervated significantly more taste buds than cluster 1 neurons. (D) Similarly, the mean ( \pm SEM) total length of axons from cluster 2 neurons was significantly greater than the axons from cluster 1 neurons, but not different from neurons in clusters 3 and 4. (E) As the number of taste buds a neuron innervated increased, the combined total length of the terminal arbors also increased (cyan=cluster 1 , magenta=cluster 2 ). The small number of cluster 2 neurons that innervated only a few taste buds (1-3), have longer terminal arbors than cluster 1 neurons. $(F)$ The average length of the terminal arbors per taste bud decreased as more taste buds are innervated. Black line indicates linear fit. ${ }^{* *} p=0.001$. Scale bars: $1 \mathrm{~mm}$ for whole nerve tracings in $A$ and $B ; 10 \mu \mathrm{m}$ for taste bud images and terminal arbor reconstructions within taste buds in $A$ and $B$.

Figure 7. An example of an axon from a cluster 3 neurons and a cluster 4 neuron. Images below each axon reconstruction show the AP staining for one taste bud innervated by this axon and the reconstructions of all the terminal arbors for this neuron. $(A)$ The representative axon of a cluster 3 neuron had a total length of $18.6 \mathrm{~mm}$, innervated 7 taste buds with 9 terminal arbors, and had 28 branch ends within the taste buds. Beneath the images are all the reconstructions of 7 additional arbors innervating 6 additional taste buds. For the two taste buds with two arbors the second arbor is shown in green. This axon also had an arbor branch innervating the epithelium outside of the taste bud (orange arrow, non-taste ending). (B) The axon of a cluster 4 neuron had a total length of $20.8 \mathrm{~mm}$, innervated 7 taste buds, and had 10 terminal arbors with total of 40 branch ends. For this example, the imaged taste bud had 4 terminal arbors (dark blue, green, red, cyan). Beneath the images are all the reconstructions of 6 additional arbors innervating 6 additional taste buds. (C-D) Plotted are medians \pm interquartile ranges (IQR (gray boxes) and \pm maximum/minimum (whiskers)). (C) The axons of cluster 3 and 4 neurons have more terminal arbors than those in clusters 1 and 2. (D) This increase in terminal arbor number is due to an 
increase in the average number of terminal arbors innervating each taste bud. (E) Across all neurons, the best indicator of the combined terminal arbor length inside the taste bud is the number of terminal arbors (blue=cluster 3 , black=cluster 4 , gray = other clusters). (F) A subset of neurons in each cluster had axons terminating outside of taste buds (medians and IQR, \pm maximum/minimum). Scale bars: $1 \mathrm{~mm}$ for whole nerve tracings in $A$ and $B ; 10 \mu \mathrm{m}$ for taste bud images and reconstructions within taste buds in $A$ and $B$.

Figure 8. Determining the number of taste-transducing cells contacting individual arbors. (A) A taste bud with all terminal arbors from taste neurons labeled magenta (tdTomato) and taste bud cells expressing PLC $\beta 2$-labeled green (sweet-, bitter-, and umami-transducing cells). The distance between nerve arbors and taste-transducing cells was measured incrementally in 1.5 voxels (110 nm, essentially overlapping). These regions were pseudo-colored white. A single section through the taste bud indicated by the white line is shown to the right of the taste bud. $A$ single magenta arbor (yellow arrowhead) contacts one PLC $\beta 2$-positive taste bud cell (green). The next closest PLC 32 -positive cell to the same arbor was pseudo-colored yellow-green is $1.2 \mu \mathrm{m}$ away (white arrow). (A, bottom) Segmenting the cells and arbors to remove florescent label outside the segmented area permits the relationship between individual arbors and taste transducing cells to be viewed. Scale bar $=5 \mu \mathrm{m}$. (B) The axons of taste neurons have numerous locations where they are within a single voxel of a taste-transducing cell. However, very few additional cells are contacted as we increase the minimum distance (in nanometers) required. As shown, labeled cells and nerve fibers tend to be either within $200 \mathrm{~nm}$ or greater than $1 \mu \mathrm{m}$ apart. This quantification was performed for all labeled nerve arbors and labeled taste-transducing cells in six taste buds (labeled with either PLC 2 [ $n=3]$ or Car4 [n=3]). (C-F) There are variations in the relationship between taste bud cells and nerve arbors. (C, E) Some arbors extend along a nerve cell for a long distance, others contact cells for shorter distances (D, F). Arbors tend to contact PLCß2-labeled cells (green) at widened regions of the arbor (C, D white arrows). Arbors tend to contact Car4-positive cells (blue) at sites of indentation of the nerve fiber into the cell (E, F, arrows). The fluorescence for each fluorophore was optimized for brightness contrast. For panels C-F complete arbors and contacted taste-transducing cells were segmented and the fluorescence outside the reconstruction removed, as illustrated in panel $A$. The reconstruction was removed and the fluorescence inside the reconstruction is shown in each panel. Scale bars in C-F are $5 \mu \mathrm{m}$ in whole cell images, and $2 \mu \mathrm{m}$ in cross-sections.

Figure 9. Number of taste-transducing cells contacted by axons from individual neurons. (A) A single axon (white arrows) is shown in three serial sections of tongue muscle (arrows). Scale bar 
$=50 \mu \mathrm{m}$. (B) Terminal arbors from three cluster 1 neurons. The axon of neuron 1 (left) innervates a single taste bud with one arbor that contacts two Car4-positive cells. The axon of neuron 2 innervates two taste buds and contacts four PLC 32 -positive cells. The axon of neuron 3 contacts a single PLC 32 -positive cell. (C) One cluster 2 neuron innervating five taste buds is shown along with the 10 taste-transducing cells contacted. All scale bars $=5 \mu \mathrm{m}$. For all panels the arbors and labeled taste bud cells that were contacted were segmented and the fluorescence outside the reconstruction was removed, as illustrated in Fig. 8A. (D) The numbers of taste-transducing cells contacted by 21 single taste axons increases across cluster. (E) Patterns in the number of arbors contacting each cell type are different across clusters, suggesting that neuron type/s likely differ across clusters. (F) When neurons are divided into groups based of the type of cells contacted, these two groups have different but overlapping distributions of combined arbor length, suggesting that neurons contacting more Car4-positive cells (sour transducing) are also more heavily branched. 


\section{Example of peripheral axon reconstruction with stained neuron segments:}

A

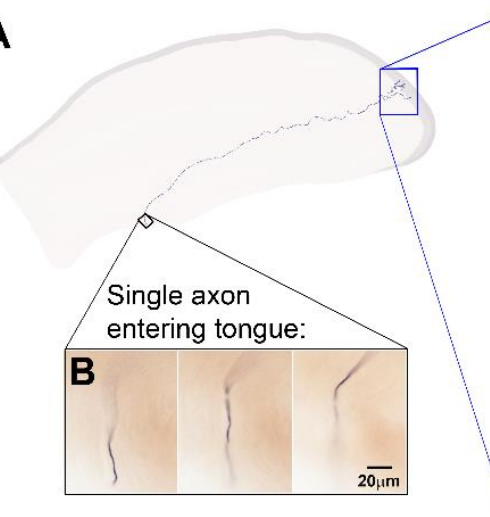

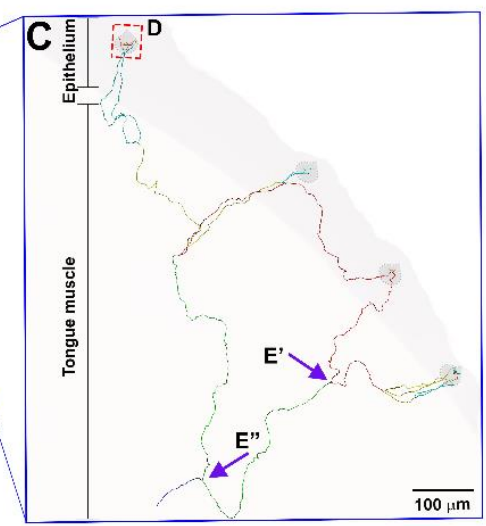

Stained arbors in taste bud:

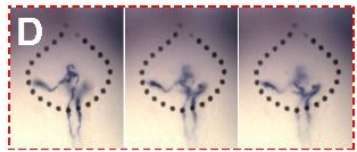

Stained branch points in tongue muscle:

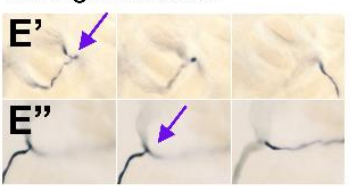

\section{Illustrations of the 16 peripheral axon measurements:}

F Whole peripheral axon measurements

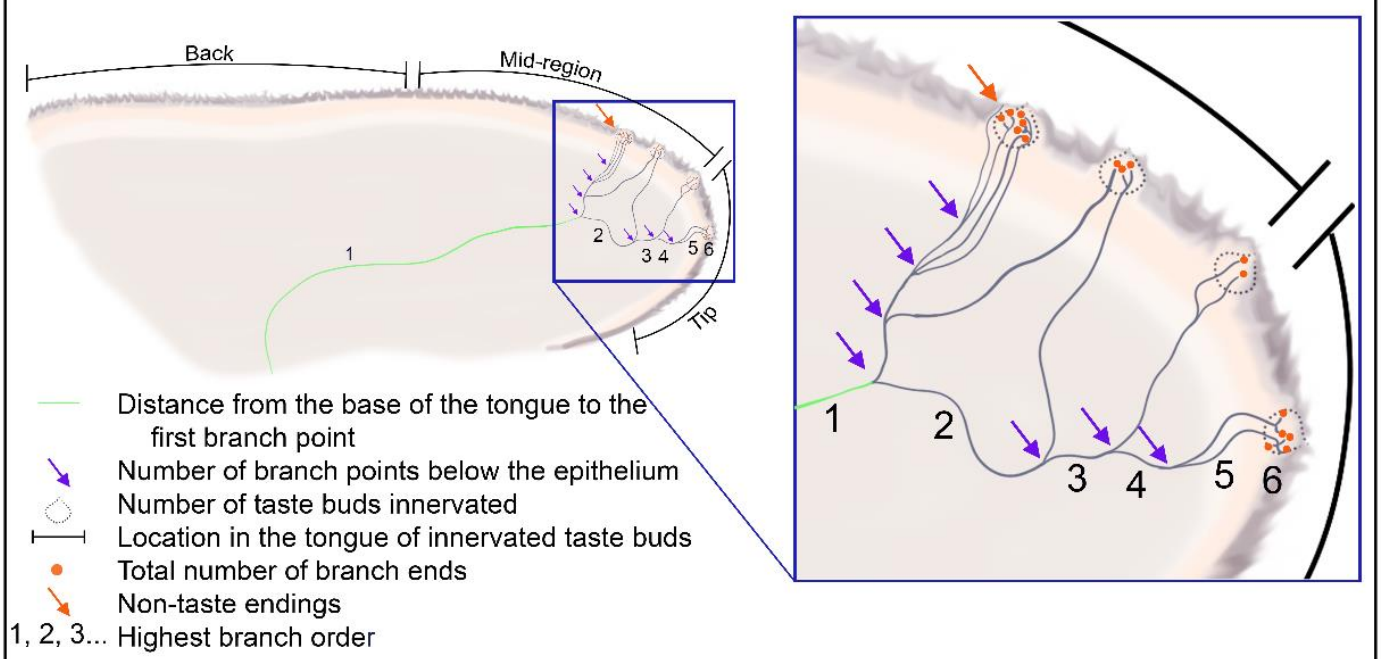

G Terminal arbor measurements

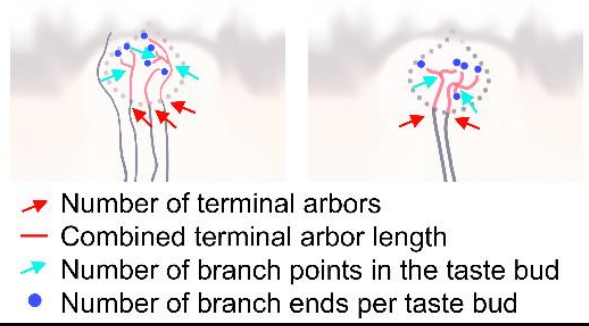

H Components of terminal arbor

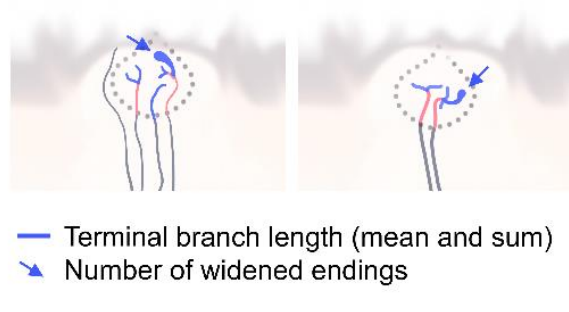

Figure 1. Genetically directed sparse-cell labeling, reconstruction, and quantification of individual taste axons. (A-E) A single AP-stained axon from a TrkB CreER:AP mouse injected with tamoxifen at postnatal day 40 . (A) A reconstruction of the taste axon starting from where it enters the tongue $(B)$ to the terminal arbors. It is superimposed on an outline of a tongue section. (B) Images of the AP-stained axon are shown at 3 different focal depths (left to right) in this $180-\mu \mathrm{m}$ section. (C) An enlarged view of the same axon with each branch segment presented in a different color. This axon innervates 4 taste buds (one of which is shown in D). Boxes illustrate the locations of branch points (E'-E'), which are each shown at 3 different focal depths. (F) illustrates 7 of the features quantified for the entire axon (in the legend), using the axon shown (A-E) as an example. In addition, the total length of the axon was measured (green and gray in F summed, $12.7 \mathrm{~mm}$ ). The location in the tongue was assigned a number (tip=1, mid-region=2, back=3); for the illustrated neuron 1.5 was assigned, since it had arbors innervated both the tip and mid-region. (G-H) Illustrate 7 additional anatomical characteristics measured in the taste buds. The scale bar in $B=20 \mu \mathrm{m}$ and applies to $\mathrm{D}$ and $\mathrm{E}$; the scale bar in $\mathrm{C}=100 \mu \mathrm{m}$. 

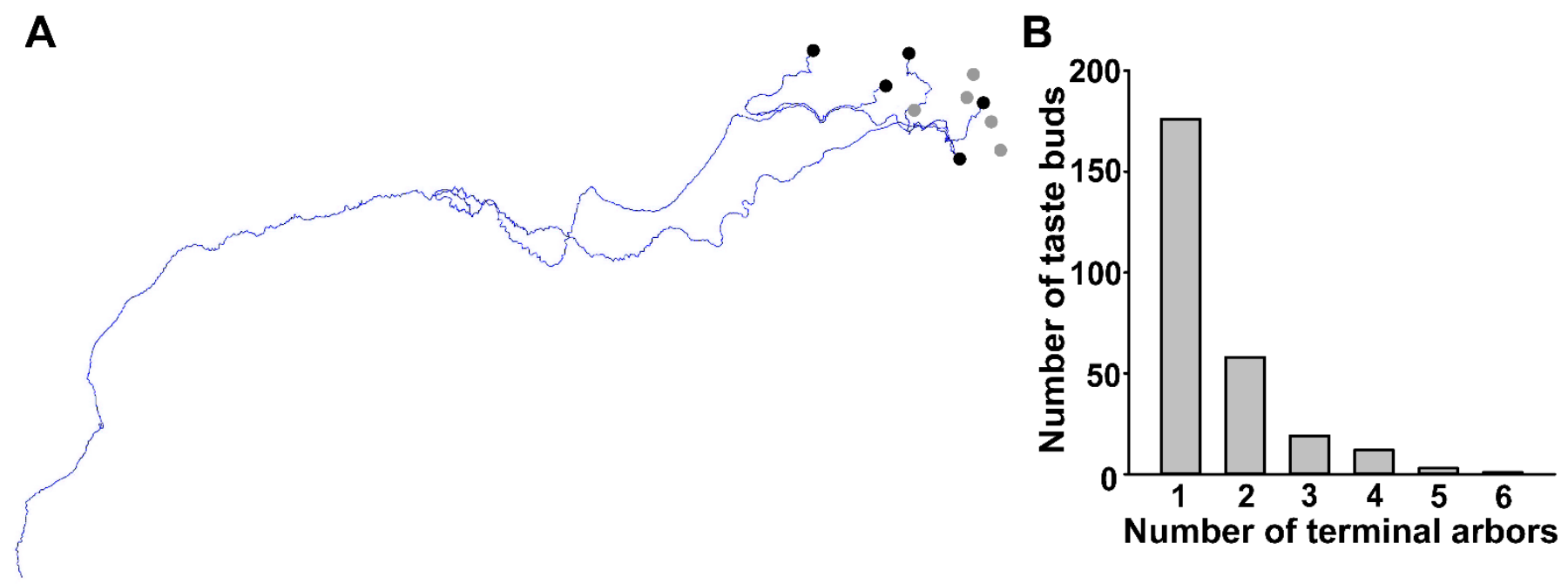

Figure 2. Anatomic receptive fields tend to be distributed over the lingual epithelial surface. (A) An example of an axon that innervated 5 taste buds, with a total of 6 terminal arbors (only one of these taste buds received more than one arbor). The fungiform papillae containing the innervated taste buds are marked by black dots. Other fungiform papillae containing taste buds not innervated by this neuron (marked by gray dots) and at least two are located between innervated papillae. Approximately $10 \%$ of axons have these extensive receptive fields. (B) A histogram illustrating the number of taste buds innervated by 1 to 6 terminal arbors from the same neuron. The 96 reconstructed axons innervate a total of 279 taste buds, and most taste buds only receive a single arbor from each taste neuron. 


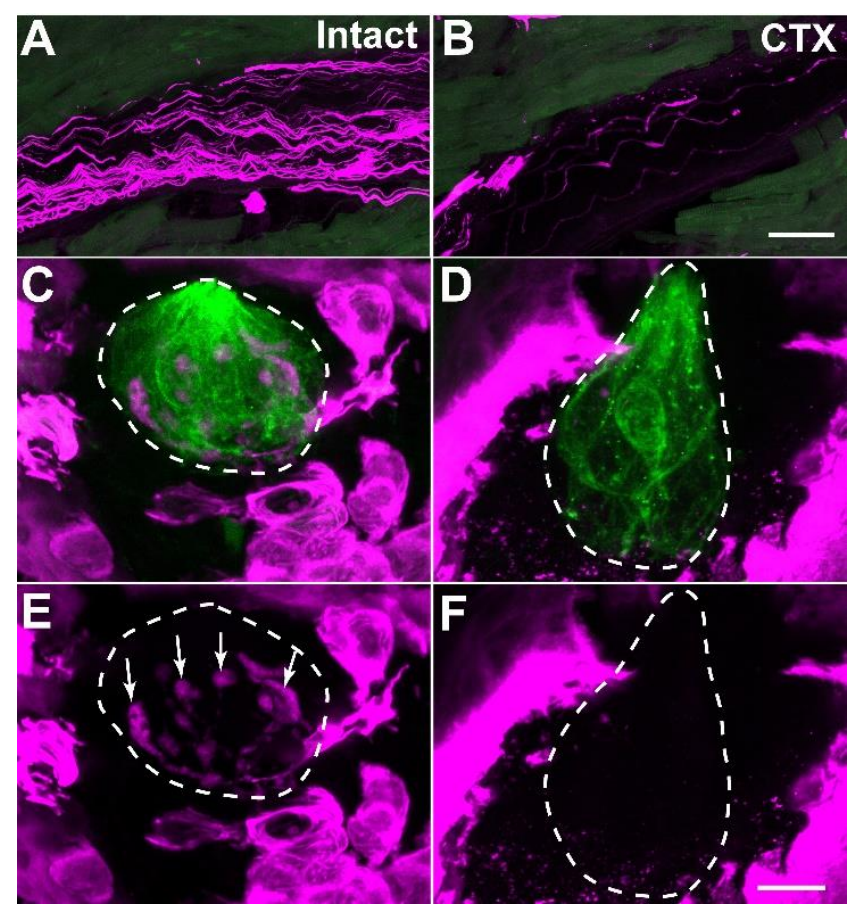

Figure 3. Most, but not all, TrkB-positive taste axons projecting to the tongue innervate taste buds. (A) On the un-transected side of the tongue (intact), abundant TrkB-positive axons (magenta) were observed in chordalingual nerve bundles. (B) On the chorda tympani transected side of the tongue (CTX) from the same mouse, substantially fewer TrkB-positive axons were observed in nerve bundles. (C) TrkB-positive axon terminals were clearly seen in taste buds labeled by cytokeratin-8 (enclosed with dashed line), from the intact side of the tongue (indicated by arrows in E). With high doses of tamoxifen label is also present in most of the non-taste epithelia around the taste bud $(\mathrm{C}-\mathrm{F}) .(\mathrm{D}, \mathrm{F})$ On the CTX side of the tongue, taste buds lacked labeled axons. Scale bar in $\mathrm{B}=50 \mu \mathrm{m}$ and also applies to $\mathrm{A}$; scale bar in $\mathrm{F}=10 \mu \mathrm{m}$ and also applies to $\mathrm{C}, \mathrm{D}$, and $\mathrm{E}$. 
A
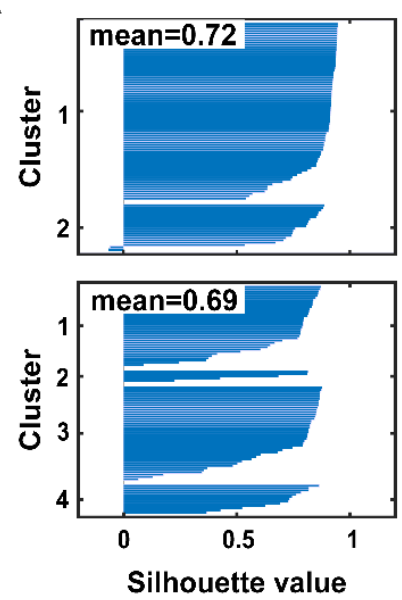

Silhouette value

B

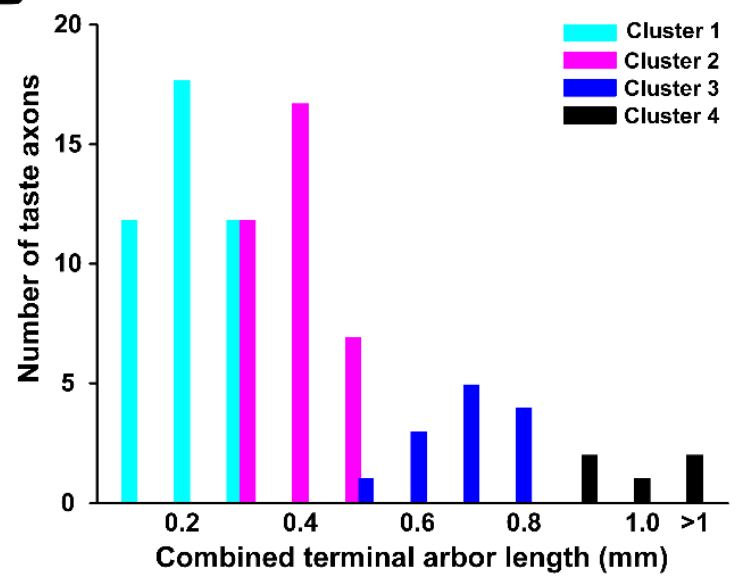

C

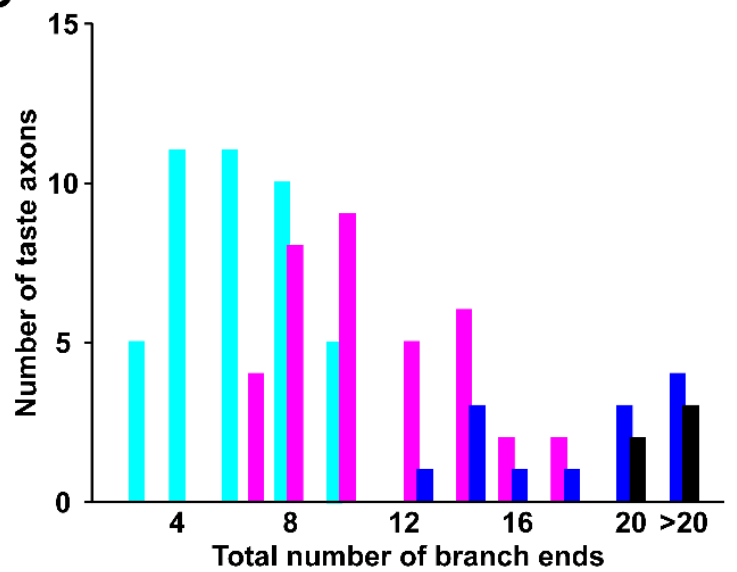

D

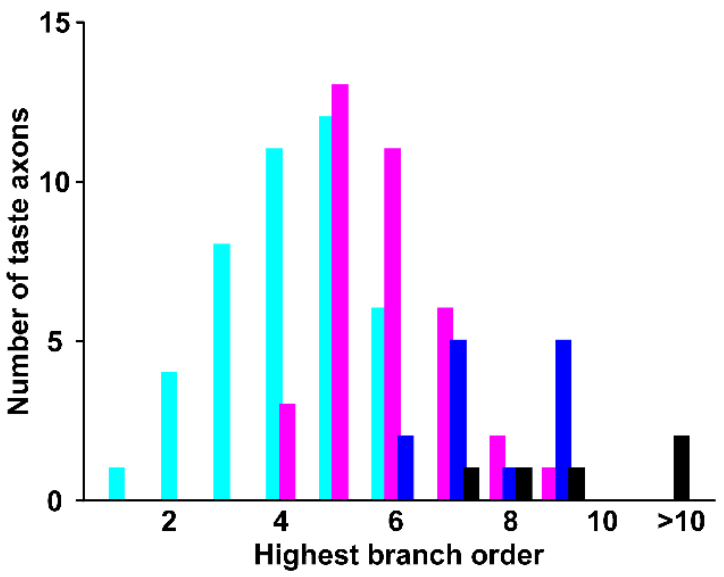

E

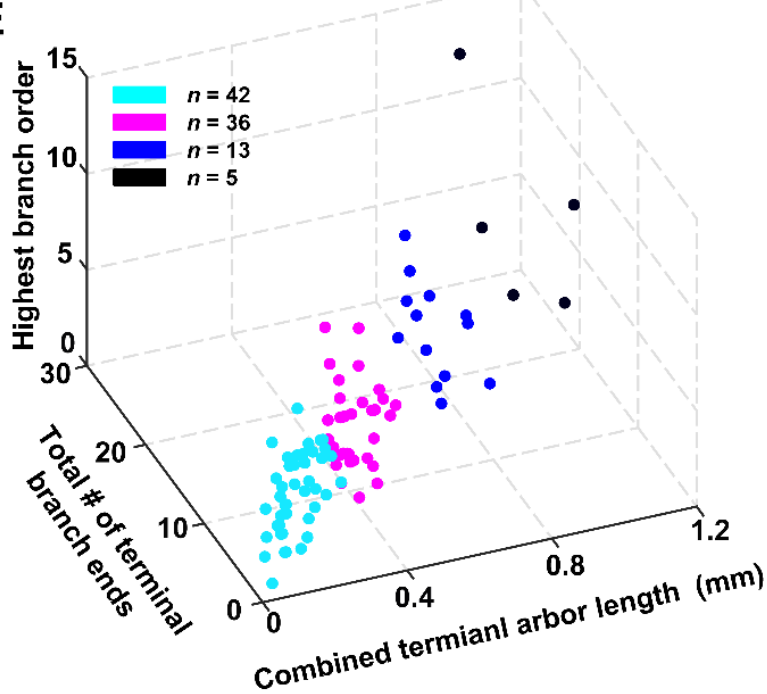

$\mathbf{F}$

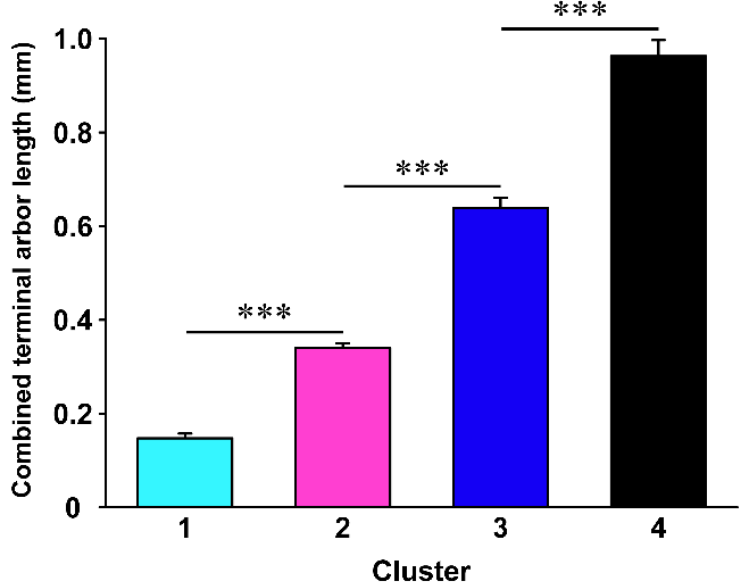

Figure 4. Taste axons were divided into four categories using a k-means cluster analysis. (A) Silhouette values are graphed for each neuron based on the similarity of each neuron to its own cluster (higher values = greater similarity). Four plots illustrate the number of clusters varying from 2 to 5 . The highest silhouette value $(0.72)$ was obtained assuming there were only two clusters, but the two-cluster model showed outliers. which also observed in the cluster 3 and the cluster 5 models. A four cluster model had the second highest mean silhouette value (0.69) with no outliers. (B-D) The distributions of combined terminal arbor length, total number of branch ends, highest branch order. These are the measures showing the greatest separation between clusters. (E) 3D graph showing the relationship of these three characteristics for individual neurons. $(F)$ Clusters are defined by combined arbor length, which is the amount of the axon available to form a connection with a taste receptor cells. ${ }^{* * *} p \leq 0.001$ 

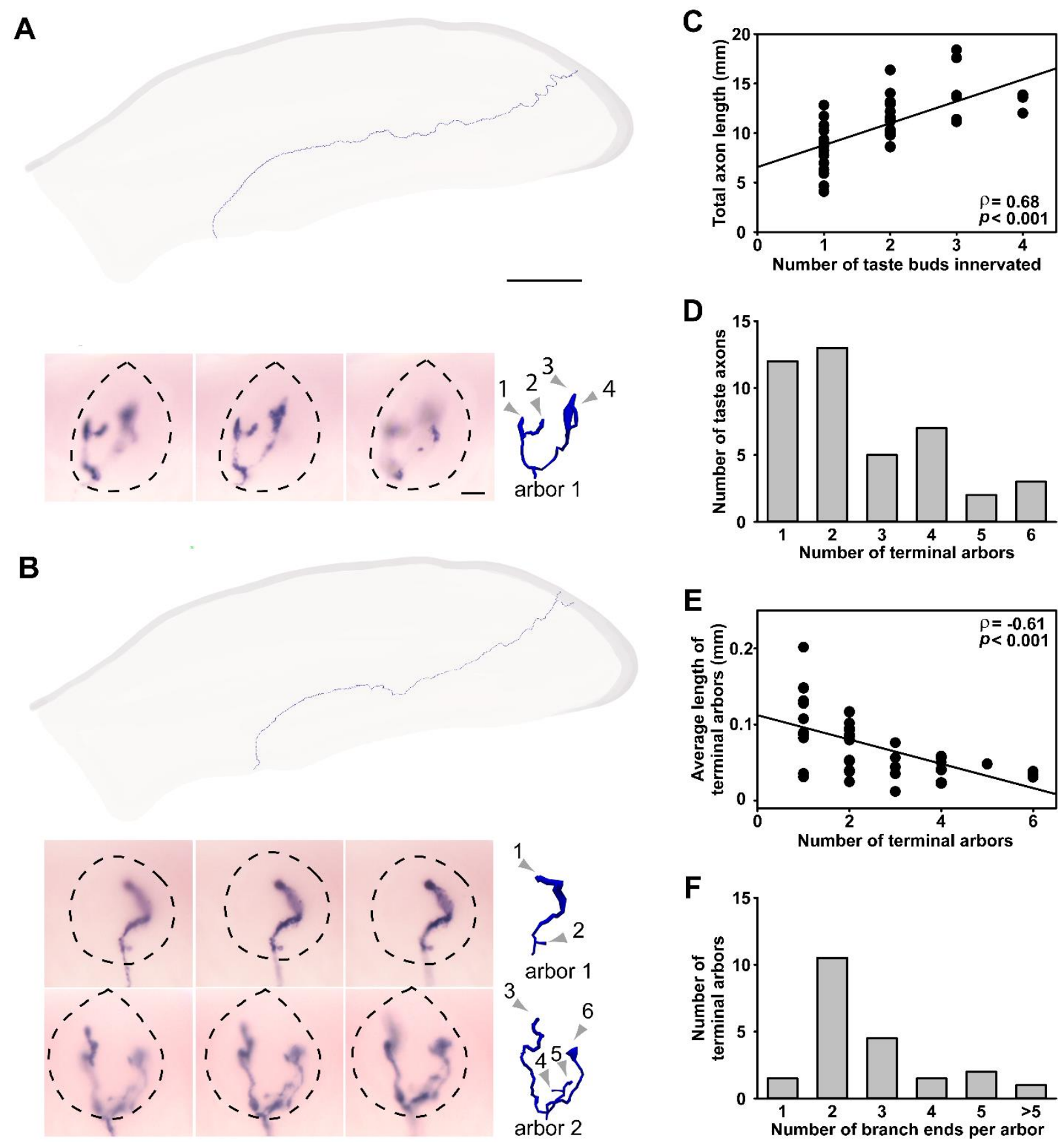

Figure 5. Two examples of axons from cluster 1 neurons. (A, B) The reconstructed axons were superimposed on an outline of one sagittal tongue section to illustrate the location of the axon in the tongue. The images below each reconstructed axon show the AP staining of terminal arbors within the taste bud at three different focal depths. Reconstructions of the terminal arbors within the taste bud are shown at the same magnification to the right of the images. (A) This 10.3-mm-long axon innervated a single taste bud with one terminal arbor, three branch points inside the taste bud, and four branch ends (gray arrowheads). (B) The second 14.0-mm-long axon innervated two taste buds with one terminal arbor each. One branch point outside the taste bud and four inside the taste bud, results in six total branch ends (gray arrowheads). (C) Axons from cluster 1 taste neurons innervate 1-4 taste buds. The number of taste buds that are innervated correlates with the total length of the axon. (D) The axons of cluster 1 neurons typically have 1 to 2 terminal arbors penetrating taste buds, but could have as many as 6 terminal arbors. (E) Axons of cluster 1 neurons with the most terminal arbors also had on average the shortest terminal arbors. (F) The distribution of the number of branch endings per terminal arbor. Most axons from cluster 1 neurons had terminal arbors with 2 branch ends. Scale bars: $1 \mathrm{~mm}$ for whole axon tracings in A and $\mathrm{B} ; 10 \mu \mathrm{m}$ for taste bud images and reconstructions within taste buds in $\mathrm{A}$ and $\mathrm{B}$. 
A
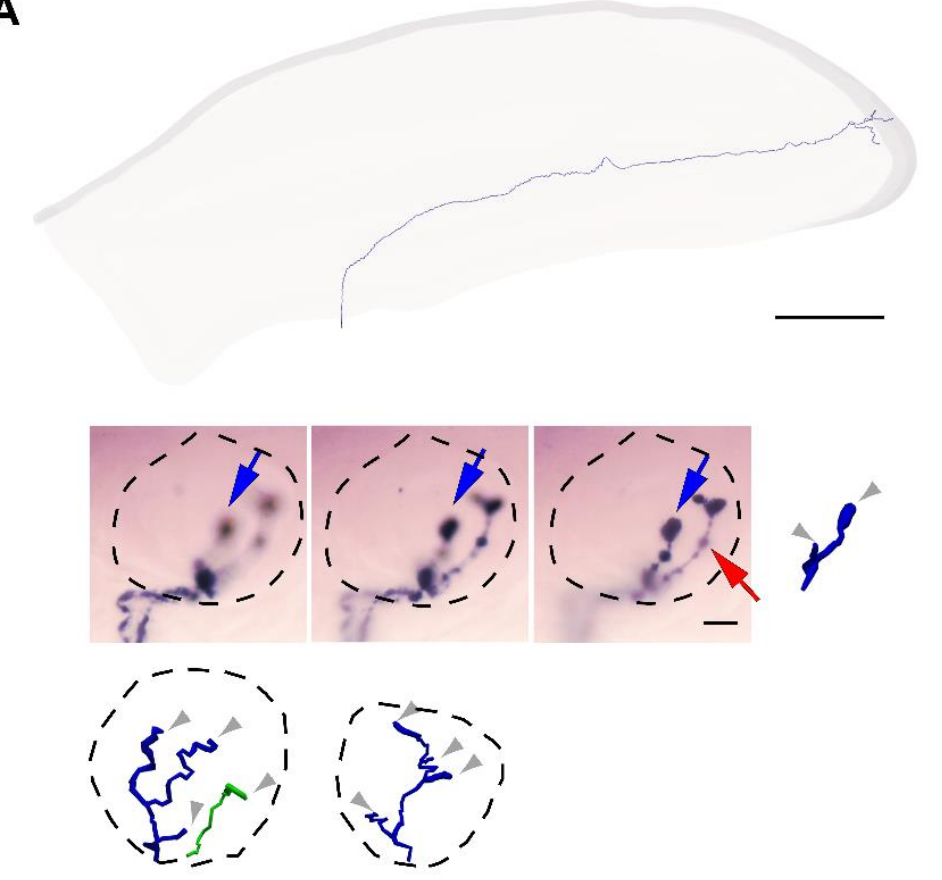

B
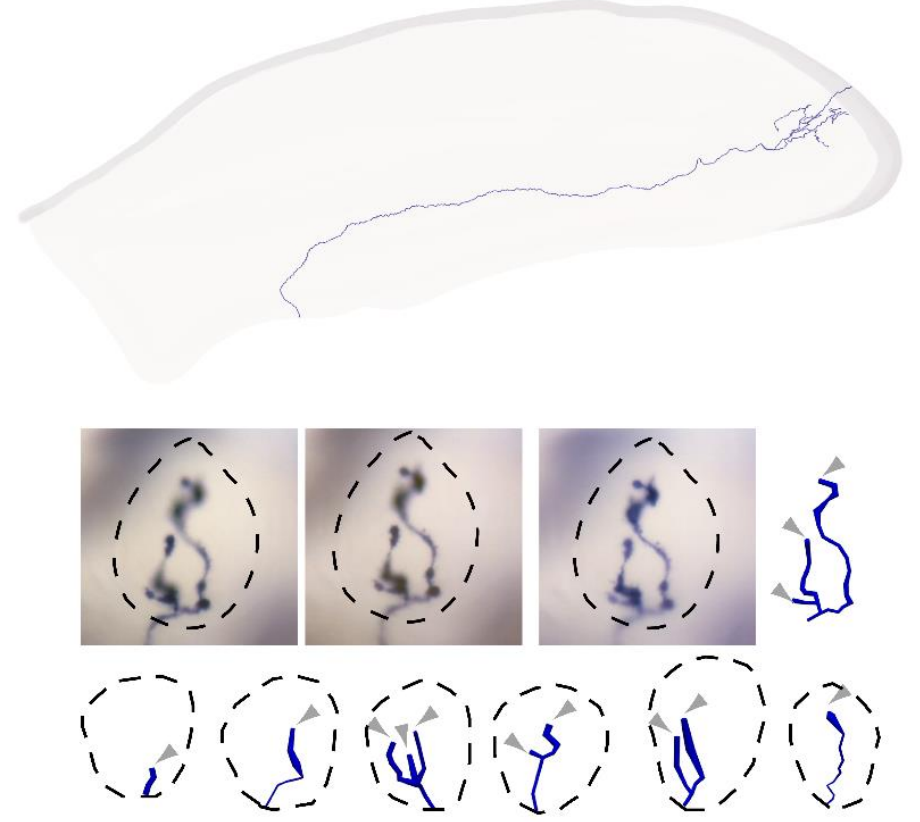

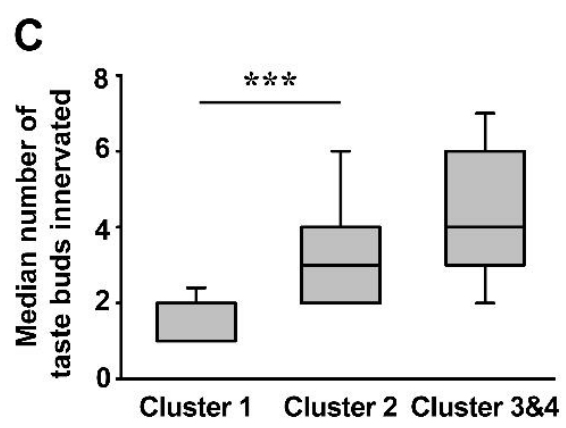

D
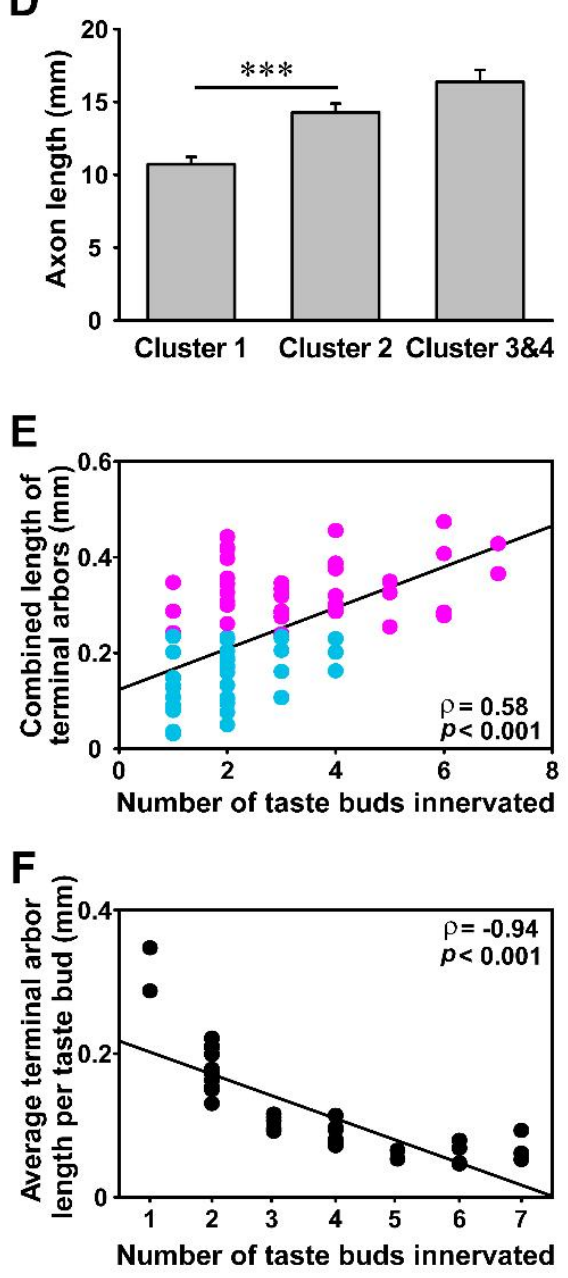

Figure 6. Two examples of axons from cluster 2 neurons. The reconstructed axons were superimposed on an outline of one sagittal tongue section to illustrate the location of the axon in the tongue. The images below each reconstructed axon show the AP staining of terminal arbors within the taste bud at three different focal depths. Reconstructions of the terminal arbors within the taste bud are shown at the same magnification to the right of the images. (A) The $13.0 \mathrm{~mm}$ axon of this cluster 2 neuron had 3 branch points below the epithelium producing 4 terminal arbors that innervated 3 taste buds (second arbor in same taste bud is green). Inside the taste bud some of the arbors had additional branch points (5) to produce 10 branch ends (gray arrowheads). The imaged taste bud has a single arbor (blue arrow) from the reconstructed axon plus one arbor from another labeled neuron (red arrow). Beneath the images are reconstructions of 3 additional terminal arbors innervating two additional taste buds. (B) The $14.7 \mathrm{~mm}$ axon from a second neuron had 6 branch points below the epithelia, producing 7 terminal arbors, each of which innervated a separate taste bud (reconstructions for 6 additional arbors are shown beneath the imaged arbor). Some of these arbors also branched in the taste bud (6 branch points) resulting in a total of 13 terminal branch ends (gray arrowheads). (C) Plots of medians \pm interquartile ranges (IQR, gray boxes) and \pm maximum/minimum (whiskers) illustrate that cluster 2 neurons innervated significantly more taste buds 
than cluster 1 neurons. (D) Similarly, the mean ( \pm SEM) total length of axons from cluster 2 neurons was significantly greater than the axons from cluster 1 neurons, but not different from neurons in clusters 3 and 4 . (E) As the number of taste buds a neuron innervated increased, the combined total length of the terminal arbors also increased (cyan=cluster 1 , magenta=cluster 2 ). The small number of cluster 2 neurons that innervated only a few taste buds (1-3), have longer terminal arbors than cluster 1 neurons. $(F)$ The average length of the terminal arbors per taste bud decreased as more taste buds are innervated. Black line indicates linear fit. ${ }^{* * *} p=0.001$. Scale bars: $1 \mathrm{~mm}$ for whole nerve tracings in $A$ and $B ; 10 \mu \mathrm{m}$ for taste bud images and terminal arbor reconstructions within taste buds in $A$ and $B$. 

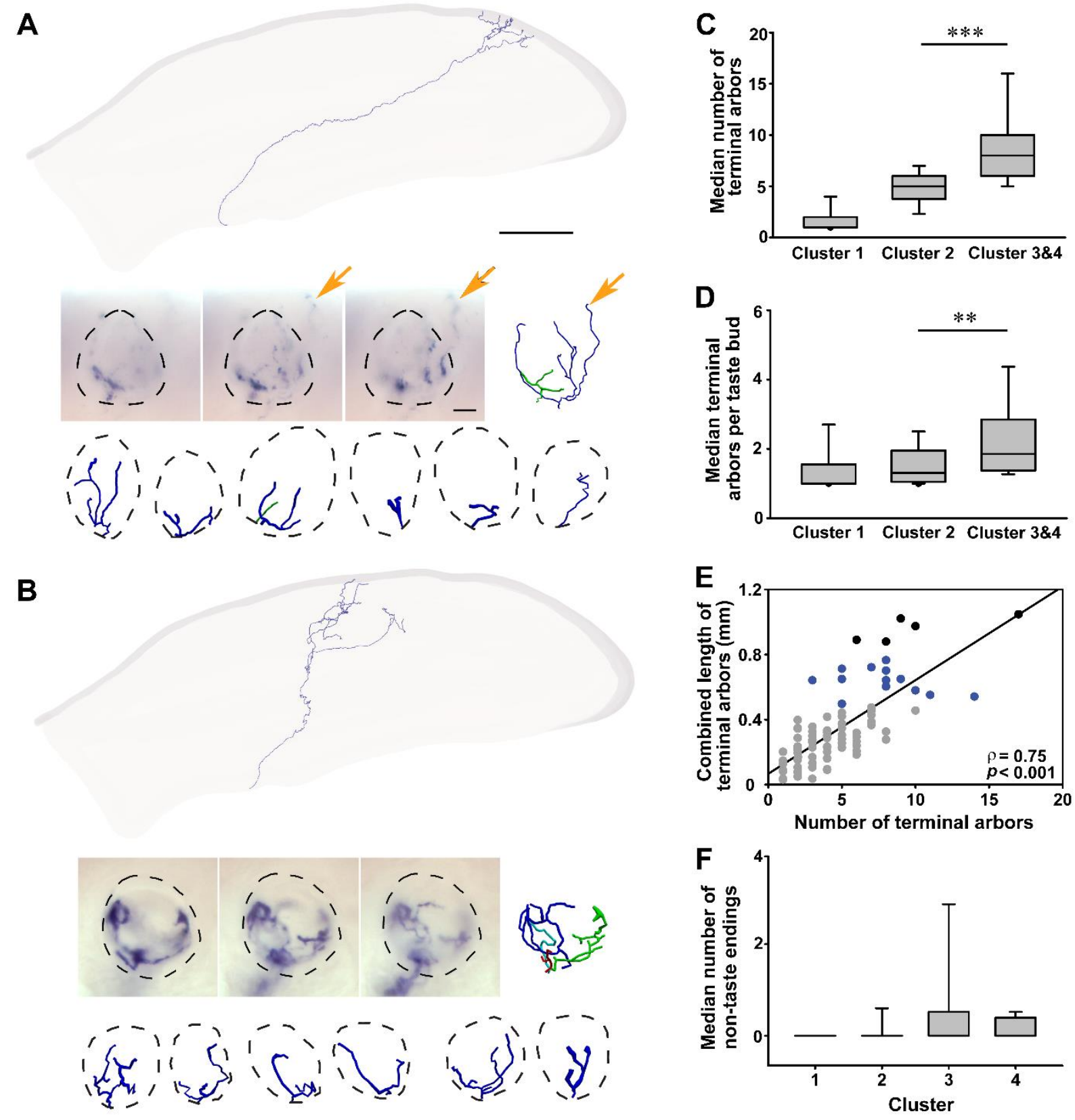

Figure 7. An example of an axon from a cluster 3 neurons and a cluster 4 neuron. Images below each axon reconstruction show the AP staining for one taste bud innervated by this axon and the reconstructions of all the terminal arbors for this neuron. (A) The representative axon of a cluster 3 neuron had a total length of $18.6 \mathrm{~mm}$, innervated 7 taste buds with 9 terminal arbors, and had 28 branch ends within the taste buds. Beneath the images are all the reconstructions of 7 additional arbors innervating 6 additional taste buds. For the two taste buds with two arbors the second arbor is shown in green. This axon also had an arbor branch innervating the epithelium outside of the taste bud (orange arrow, non-taste ending). (B) The axon of a cluster 4 neuron had a total length of $20.8 \mathrm{~mm}$, innervated 7 taste buds, and had 10 terminal arbors with total of 40 branch ends. For this example, the imaged taste bud had 4 terminal arbors (dark blue, green, red, cyan). Beneath the images are all the reconstructions of 6 additional arbors innervating 6 additional taste buds. (C-D) Plotted are medians \pm interquartile ranges (IQR (gray boxes) and \pm maximum/minimum (whiskers)). (C) The axons of cluster 3 and 4 neurons have more terminal arbors than those in clusters 1 and 2. (D) This increase in terminal arbor number is due to an increase in the average number of terminal arbors innervating each taste bud. (E) Across all neurons, the best indicator of the combined terminal arbor length inside the taste bud is the number of terminal arbors (blue=cluster 3 , black=cluster 4 , gray = other clusters). (F) A subset of neurons in each cluster had axons terminating outside of taste buds (medians and IQR, \pm maximum/minimum). Scale bars: $1 \mathrm{~mm}$ for whole nerve tracings in $A$ and $B ; 10 \mu \mathrm{m}$ for taste bud images and reconstructions within taste buds in $\mathrm{A}$ and $\mathrm{B}$. 

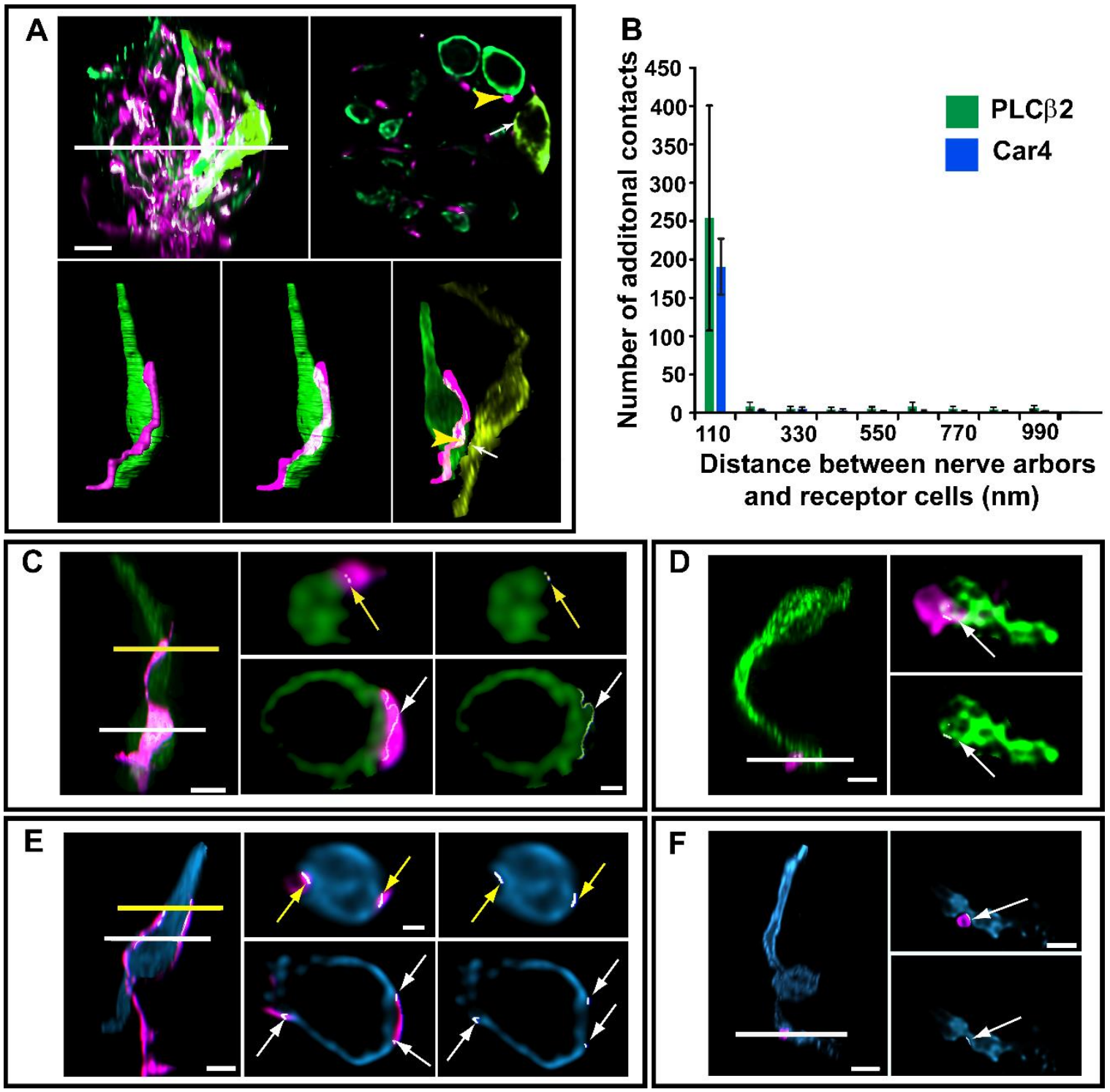

Figure 8. Determining the number of taste-transducing cells contacting individual arbors. (A) A taste bud with all terminal arbors from taste neurons labeled magenta (tdTomato) and taste bud cells expressing PLC 32 -labeled green (sweet-, bitter-, and umami-transducing cells). The distance between nerve arbors and taste-transducing cells was measured incrementally in 1.5 voxels $(110 \mathrm{~nm}$, essentially overlapping). These regions were pseudocolored white. A single section through the taste bud indicated by the white line is shown to the right of the taste bud. A single magenta arbor (yellow arrowhead) contacts one PLCß2-positive taste bud cell (green). The next closest PLC $\beta 2$-positive cell to the same arbor was pseudo-colored yellow-green is $1.2 \mu \mathrm{m}$ away (white arrow). (A, bottom) Segmenting the cells and arbors to remove florescent label outside the segmented area permits the relationship between individual arbors and taste transducing cells to be viewed. Scale bar $=5 \mu \mathrm{m}$. $(\mathrm{B})$ The axons of taste neurons have numerous locations where they are within a single voxel of a taste-transducing cell. However, very few additional cells are contacted as we increase the minimum distance (in nanometers) required. As shown, labeled cells and nerve fibers tend to be either within $200 \mathrm{~nm}$ or greater than $1 \mu \mathrm{m}$ apart. This quantification was performed for all labeled nerve arbors and labeled taste-transducing cells in six taste buds (labeled with either PLC 2 [ $n=3]$ or Car4 $[n=3])$. (C-F) There are variations in the relationship between taste bud cells and nerve arbors. (C, E) Some arbors extend along a nerve cell for a long distance, others contact cells for shorter distances (D, F). Arbors tend to contact PLCß2-labeled cells (green) at widened regions of the arbor (C, $\mathrm{D}$ white arrows). Arbors tend to contact Car4-positive cells (blue) at sites of indentation of the nerve fiber into the cell (E, F, arrows). The fluorescence for each fluorophore was optimized for brightness contrast. For panels CF complete arbors and contacted taste-transducing cells were segmented and the fluorescence outside the reconstruction removed, as illustrated in panel $A$. The reconstruction was removed and the fluorescence inside the reconstruction is shown in each panel. Scale bars in C-F are $5 \mu \mathrm{m}$ in whole cell images, and $2 \mu \mathrm{m}$ in crosssections. 

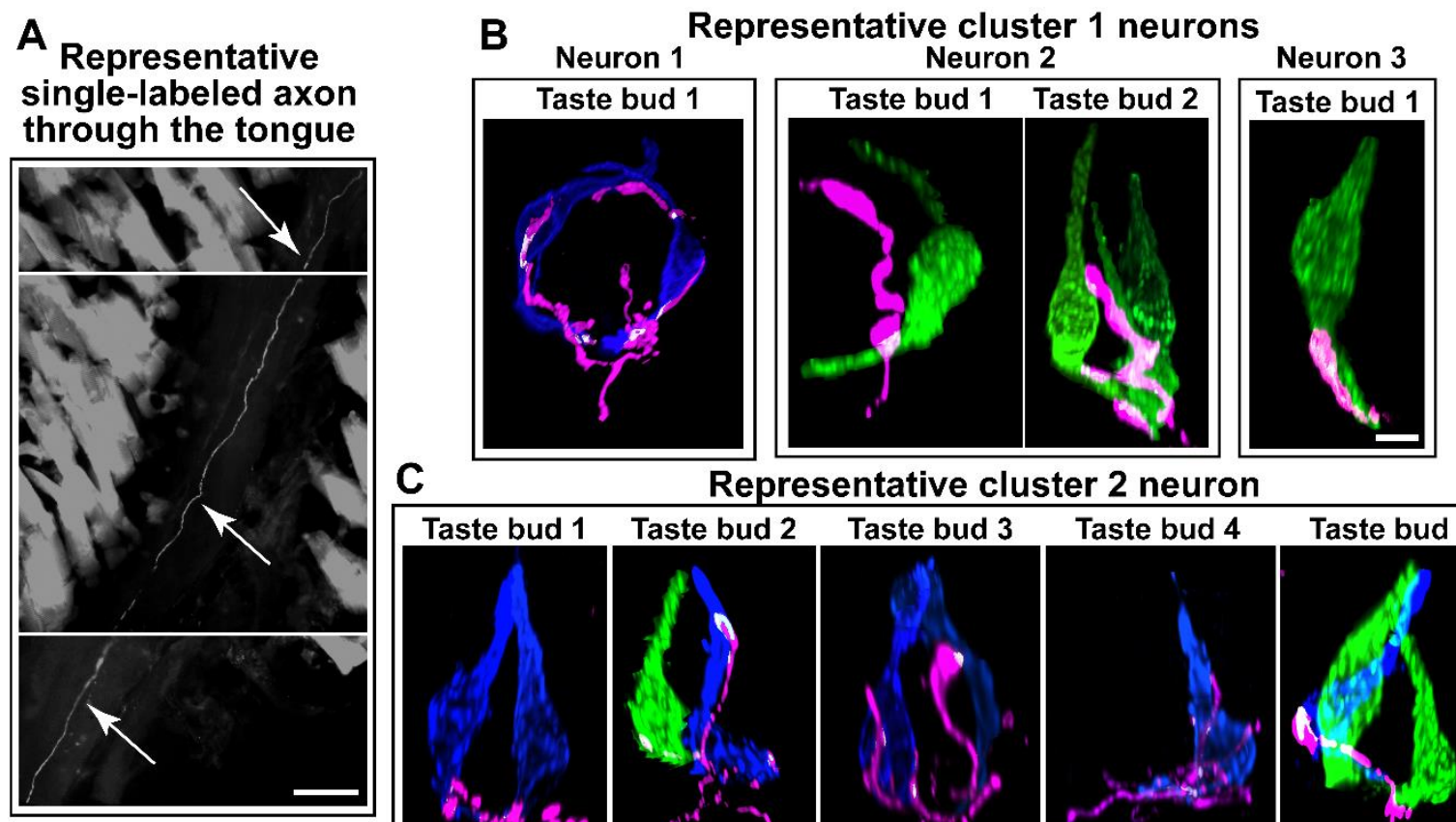

C

Representative cluster 2 neuron
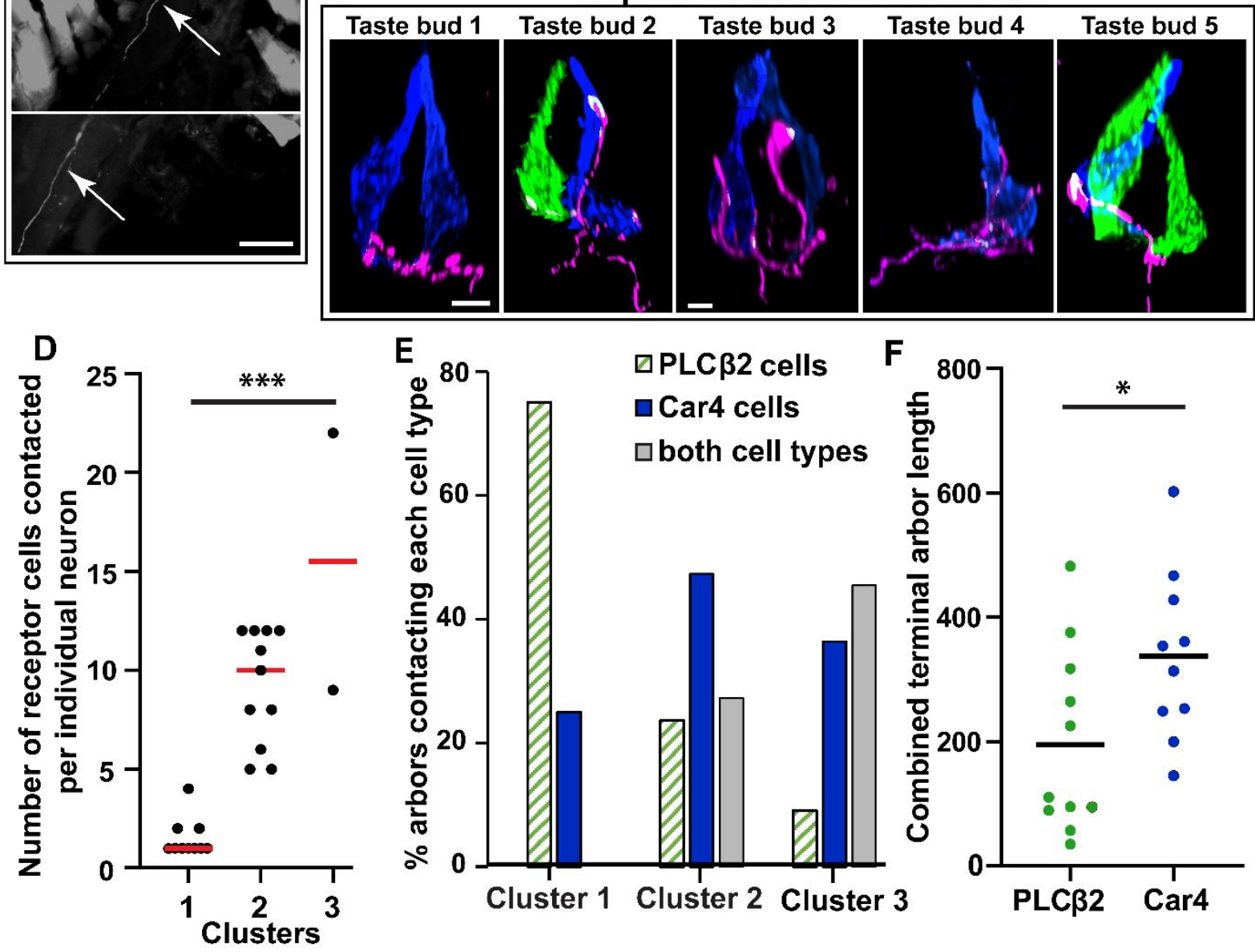

Figure 9. Number of taste-transducing cells contacted by axons from individual neurons. (A) A single axon (white arrows) is shown in three serial sections of tongue muscle (arrows). Scale bar $=50 \mu \mathrm{m}$. (B) Terminal arbors from three cluster 1 neurons. The axon of neuron 1 (left) innervates a single taste bud with one arbor that contacts two Car4-positive cells. The axon of neuron 2 innervates two taste buds and contacts four PLC 32 -positive cells. The axon of neuron 3 contacts a single PLCß2-positive cell. (C) One cluster 2 neuron innervating five taste buds is shown along with the 10 taste-transducing cells contacted. All scale bars $=5 \mu \mathrm{m}$. For all panels the arbors and labeled taste bud cells that were contacted were segmented and the fluorescence outside the reconstruction was removed, as illustrated in Fig. 8A. (D) The numbers of taste-transducing cells contacted by 21 single taste axons increases across cluster. (E) Patterns in the number of arbors contacting each cell type are different across clusters, suggesting that neuron type/s likely differ across clusters. (F) When neurons are divided into groups based of the type of cells contacted, these two groups have different but overlapping distributions of combined arbor length, suggesting that neurons contacting more Car4-positive cells (sour transducing) are also more heavily branched. 